Alex Gamburd · Dmitry Jakobson · Peter Sarnak

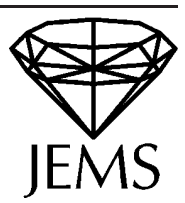

\title{
Spectra of elements in the group ring of SU(2)
}

Received June 1, 1998 / final version received September 8, 1998

Abstract. We present a new method for establishing the "gap" property for finitely generated subgroups of SU(2), providing an elementary solution of Ruziewicz problem on $S^{2}$ as well as giving many new examples of finitely generated subgroups of SU(2) with an explicit gap. The distribution of the eigenvalues of the elements of the group ring $\mathbf{R}[\mathrm{SU}(2)]$ in the $N$-th irreducible representation of SU(2) is also studied. Numerical experiments indicate that for a generic (in measure) element of $\mathbf{R}[\mathrm{SU}(2)$ ], the "unfolded" consecutive spacings distribution approaches the GOE spacing law of random matrix theory (for $N$ even) and the GSE spacing law (for $N$ odd) as $N \rightarrow \infty$; we establish several results in this direction. For certain special "arithmetic" (or Ramanujan) elements of R[SU(2)] the experiments indicate that the unfolded consecutive spacing distribution follows Poisson statistics; we provide a sharp estimate in that direction.

\section{Introduction}

The irreducible representations of $G=\mathrm{SU}(2)$ are

$$
\pi_{N}=\operatorname{sym}^{N} V, \quad N \geq 0,
$$

where $V$ is the standard two-dimensional representation of $G$. The dimension of $\pi_{N}$ is $N+1$ and it may be realized concretely by the linear action

$$
(x, y) \rightarrow(\alpha x+\gamma y, \beta x+\delta y), \quad\left[\begin{array}{ll}
\alpha & \beta \\
\gamma & \delta
\end{array}\right] \in G
$$

on $W_{N+1}$, the space of homogeneous polynomials in $(x, y)$ of degree $N$. The character $\chi_{N}$ of $\pi_{N}$ at

$$
g=\left[\begin{array}{cc}
e^{i \alpha} & 0 \\
0 & e^{-i \alpha}
\end{array}\right] \text { is } \frac{\sin (N+1) \alpha}{\sin \alpha} .
$$

A. Gamburd, P. Sarnak: Department of Mathematics, Princeton University, Princeton, NJ 08544, USA

D. Jakobson: IAS, School of Mathematics, Princeton, NJ 08540 and Mathematics, 253-37, Caltech, Pasadena, CA 91125, USA

A. G. was supported by the NSF graduate fellowship, D. J. was supported by the NSF postdoctoral fellowship and P. S. by the NSF grant DMS-9401571

Mathematics Subject Classification (1991): 11, 22E45, 42Axx, 54H15, 81Q50 
This well known character formula is our main tool for studying the spectra of the elements in the group ring, $\mathbf{R}[G]$. Let $z$ be such an element,

$$
z=\sum_{g \in G} z_{g} g
$$

with $z_{g} \in \mathbf{R}$ and $z_{g}=0$ for all but a finite number of $g \in G$. Let $\operatorname{supp}(z)=$ $\left\{g \in G \mid z_{g} \neq 0\right\}$ and $\Gamma_{z}$ the group generated by $\operatorname{supp}(z)$. Clearly $\Gamma_{z}$ is a finitely generated subgroup of $G$.

For $\pi=\pi_{N}$ as above let $\widehat{z}(\pi)$ be the $(N+1) \times(N+1)$ matrix

$$
\widehat{z}(\pi)=\sum_{g \in G} z_{g} \pi(g)
$$

We are interested in the spectrum, $\operatorname{spec}\left(\widehat{z}\left(\pi_{N}\right)\right)$, of $\widehat{z}\left(\pi_{N}\right)$ (which of course does not depend on the realization of $\left.\pi_{N}\right)$ as $N \rightarrow \infty$. This question is of interest in analysis (for example the Ruziewicz problem see [CV,LPS,Lub], and "strongly ergodic actions" [Sch]) as well as in the theory of quantization ("quantum chaos" [Sa2,Zel2]). The connection to the latter comes from $\Gamma_{z}$ acting on $S^{2}$ (using the double cover $\mathrm{SU}(2) \rightarrow \mathrm{SO}(3)$ ) as symplectic maps and an $N$-th quantization (" $N=1 / h$ ") of this classical action being realized by $\widehat{z}\left(\pi_{N}\right)$ [Zel1]. According to general philosophies in quantization theory [BGS] one might expect that if $\Gamma_{z}$ acts ergodically on $S^{2}, \operatorname{spec}\left(\widehat{z}\left(\pi_{N}\right)\right)$ behaves like the spectrum of a typical member of an appropriate ensemble of random matrices (cf. [M]). With suitable interpretations, this expectation is borne out rather well in the numerical experiments described in Sect. 5. This appears to be one of the simplest examples of this phenomenon.

We assume further that $z$ is self-adjoint, i.e. $z^{*}=\sum \bar{z}_{g} g^{-1}=z$. In this case $\widehat{z}\left(\pi_{N}\right)$ is self-adjoint with respect to a suitable inner product on $W_{N+1}$. Hence $\operatorname{spec}\left(\widehat{z}\left(\pi_{N}\right)\right)$ is real and is contained in the interval $[-|| z||,|| z||]$, where $\|z\|=\sum_{g \in G}\left|z_{g}\right|$. Let ||$\widehat{z}\left(\pi_{N}\right) \|$ be the norm of the matrix $\widehat{z}\left(\pi_{N}\right)$ with respect to the above inner product. ||$\widehat{z}\left(\pi_{N}\right) \|$ is equal to the maximum of $\left|\lambda_{j}\left(\widehat{z}\left(\pi_{N}\right)\right)\right|$ where

$$
\lambda_{0}\left(\widehat{z}\left(\pi_{N}\right)\right) \geq \lambda_{1}\left(\widehat{z}\left(\pi_{N}\right)\right) \geq \ldots \geq \lambda_{N}\left(\widehat{z}\left(\pi_{N}\right)\right)
$$

are the eigenvalues of $\widehat{z}\left(\pi_{N}\right)$.

A fundamental question for a positive $z$ (i.e. $z_{g} \geq 0$ for $g \in G$ ) is whether it has a gap by which we mean that

$$
\varlimsup_{N \rightarrow \infty}\left\|\widehat{z}\left(\pi_{N}\right)\right\|<\|z\| .
$$

Constructing any $z$ with a gap is apparently rather difficult and was first achieved by Drinfeld [Dri]. It was used by him to give the final step in 
the resolution of Ruziewicz problem asserting that the Haar measure $S^{2}$ is the unique finitely additive rotation invariant measure defined on Lebesgue sets. Drinfeld's method appeals to some sophisticated machinery from the theory of automorphic representations (namely the Jacquet Langlands correspondence and Deligne's solution of the Ramanujan conjectures). One of our aims here is to give a new elementary and perhaps more importantly robust analytic method for establishing that certain $z$ 's have a gap. In particular we construct new $z$ 's with this property and thus in passing provide an elementary analytic solution of the Ruziewicz problem. The discussion in Sect. 3 reduces the issue of a gap to estimating the number of elements in $\Gamma_{z}$ (ordered suitably with word length) with trace near 2 . This estimation can be carried out easily for $z$ 's constructed via integers, on the other hand doing so for the case of "generic" $z$ (see below) has thus far eluded us. So let $H(\mathbf{Z})$ denote the ring of Hamilton quaternions $\alpha=x_{0}+x_{1} i+x_{2} j+x_{3} k, x_{j} \in \mathbf{Z}$. Let $\bar{\alpha}=x_{0}-x_{1} i-x_{2} j-x_{3} k$ and $N(\alpha)=\alpha \bar{\alpha}$. For $q \geq 3$ a prime number let $\tilde{g}_{1}, \tilde{g}_{2}, \ldots, \tilde{g}_{k}$ be a subset of $S=\{\alpha \in H(\mathbf{Z}) \mid N(\alpha)=q\}$ (it is well known [HW] that the latter has $8(q+1)$ elements) satisfying

(I) $\tilde{g}_{j_{1}} \neq \varepsilon \tilde{g}_{j_{2}}$ for $j_{1} \neq j_{2}$ and $\varepsilon \in\{ \pm 1, \pm i, \pm j, \pm k\}$ a unit.

(II) $\tilde{g}_{j_{1}} \neq \varepsilon \overline{\tilde{g}_{j_{2}}}$ for any $j_{1}, j_{2}$ and $\varepsilon$ a unit.

The homomorphism of $H(\mathbf{R})$ into SU(2)

$$
\alpha \rightarrow \frac{1}{\sqrt{N(\alpha)}}\left[\begin{array}{cc}
x_{0}+x_{1} i & x_{2}+x_{3} i \\
-x_{2}+x_{3} i & x_{0}-x_{1} i
\end{array}\right]
$$

gives us the corresponding elements $g_{1}, g_{2}, \ldots, g_{k} \in G$.

Theorem 1.1. Let $q \geq 3, g_{1}, g_{2}, \ldots, g_{k} \in G$ be as above. If $p=2 k-1>$ $q^{4 / 5}$ then $z=g_{1}+g_{1}^{-1}+\ldots+g_{k}+g_{k}^{-1}$ has a gap, in fact

$$
\varlimsup_{N \rightarrow \infty}\left\|\widehat{z}\left(\pi_{N}\right)\right\| \leq p^{1 / 2}\left(\frac{q^{2 / 3}}{p^{1 / 3}}+\frac{p^{1 / 3}}{q^{2 / 3}}\right)<2 k
$$

For example if $q=7$,

$$
\tilde{g}_{1}=2-i+j+k, \quad \tilde{g}_{2}=2-i-j+k, \tilde{g}_{3}=2+i-j+k
$$

satisfy the hypotheses and denoting the corresponding $z$ by $\tilde{z}_{7}$ we have

$$
\varlimsup_{N \rightarrow \infty}\left\|\widehat{\tilde{z}}_{7}\left(\pi_{N}\right)\right\| \leq 5.83(<6) .
$$

Remark 1.2.

(1) If $q \equiv 1(\bmod 4)$ and we choose a maximal such subset $\tilde{g}_{1}, \ldots, \tilde{g}_{k}$ of $S$ above, then $2 k-1=q$ and we get an element $z$ which we denote $z_{q}$ which according to the above theorem satisfies

$$
\varlimsup_{N \rightarrow \infty}\left\|\widehat{z_{q}}\left(\pi_{N}\right)\right\| \leq q^{1 / 2}\left(q^{1 / 3}+q^{-1 / 3}\right)<q+1
$$


For this element it was shown in [LPS] using automorphic forms and in particular Deligne's proof of the Ramanujan Conjectures that

$$
\left\|\widehat{z_{q}}\left(\pi_{N}\right)\right\| \leq 2 \sqrt{q} \quad \text { for all } N \geq 1 \text {. }
$$

This bound is optimal (see Sect. 4). We call a general element $z$ of the form $g_{1}+g_{1}^{-1}+\ldots+g_{k}+g_{k}^{-1}($ where $k \geq 2)$ for which $\left\|\widehat{z}\left(\pi_{N}\right)\right\| \leq$ $2 \sqrt{2 k-1}$ holds when $N \geq 1$, a Ramanujan element.

(2) For $z \neq z_{q}$ as above (i.e. $p<q$ ) the existing methods from automorphic forms do not, as far as we know, apply to show that $z$ has a gap. In more detail, it is clear that in general $z$ having a gap is a property of $\Gamma_{z}$. The method of Drinfeld [Dri] for establishing a gap ensures that if $\Gamma_{z}$ contains an arithmetic group then $z$ has a gap (by an arithmetic subgroup $\Lambda$ of $G$ we mean a group obtained from a quaternion algebra as follows; let $K / \mathbf{Q}$ be a number field and $D / K$ a quaternion algebra. Assume that $K$ has an archimedian place $v$ such that $D \otimes K_{v}$ is the Hamiltonian quaternions. In particular $D^{(1)} \otimes K_{v}$, where the super 1 denotes elements of norm 1, is isomorphic to $\mathrm{SU}(2)$. Let $\mathcal{P}$ be a (nonempty) set of places containing the other archimedian places of $K$ and let $\Gamma$ be the group of elements of $D^{(1)}(K)$ which are integral outside $\mathcal{P}$ and let $\Gamma^{v}$ be the projection of $\Gamma$ in $D^{(1)} \otimes K_{v} \simeq \mathrm{SU}(2)$. We call $\Lambda \subset \mathrm{SU}(2)$ arithmetic if it is commensurable with a conjugate of such a $\Gamma^{v}$ ). For $p<q, \Gamma_{z}$ does not contain an arithmetic group. The element $\tilde{z}_{7}$ is an example of the above and as numerical computation shows, see Sect. 5, it is not Ramanujan. We remark that Shalom [Sha] has given (in another context) $\Gamma$-s with the analogue of a gap, which are constructed as commutator subgroups of arithmetic groups.

Next we discuss the distribution of the eigenvalues (5) as $N \rightarrow \infty$. This requires distinguishing the case $N$ even and $N$ odd, since the corresponding $\pi_{N}$ has a different symmetry in each case. As is well known and discussed in Sect. 2, for $N$ even $\pi_{N}$ preserves a symmetric bilinear form on $W_{N+1}$ while for $N$ odd it preserves a skew-symmetric form. It follows that with suitable bases for $W_{N+1}$, for $N$ even $\widehat{z}\left(\pi_{N}\right)$ lies in $\mathcal{H}_{N+1}$ the (real) linear space of $(N+1) \times(N+1)$ real-symmetric matrices, while for $N$ odd $\widehat{z}\left(\pi_{N}\right)$ lies in the (real) linear space, also denoted $\mathcal{H}_{N+1}$, of $(N+1) \times(N+1)$ matrices $H$ satisfying

$$
H^{*}=H, \quad J^{t} H J=H^{t}, J=\left[\begin{array}{cccc}
E & 0 & \ldots & 0 \\
0 & E & \ldots & 0 \\
\vdots & \vdots & \ddots & \vdots \\
0 & 0 & \ldots & E
\end{array}\right], E=\left[\begin{array}{cc}
0 & 1 \\
-1 & 0
\end{array}\right]
$$


In particular in this latter case the eigenvalues of $\widehat{z}\left(\pi_{N}\right)$ are of the form $\lambda_{1}, \lambda_{2}, \ldots, \lambda_{M}$ where $M=(N+1) / 2$ with each $\lambda_{j}$ occuring with multiplicity 2 .

While our main interest lies in $\operatorname{spec}\left(\widehat{z}\left(\pi_{N}\right)\right)$ as $N \rightarrow \infty$ we prove in Sect. 2, see Proposition 2.1, that if

$$
z=g_{1}+g_{1}^{-1}+\ldots+g_{k}+g_{k}^{-1}
$$

with $\left(g_{1}, \ldots, g_{k}\right) \in G^{(k)}$ chosen at random according to Haar measure, and if $k$ and $N$ both go to infinity then local statistics of the eigenvalues of $\widehat{z}\left(\pi_{N}\right)$ follow GOE statistics for $N$ even and GSE statistics for $N$ odd (see Sect. 2 for the definitions of these ensembles).

Returning to $z$ fixed and $N \rightarrow \infty$ we examine the densities of the eigenvalues of $\widehat{z}\left(\pi_{N}\right)$. These are described by the sum of point masses

$$
\mu_{N}(z)=\frac{1}{N+1} \sum_{j=0}^{N} \delta_{\lambda_{j}\left(\widehat{z}\left(\pi_{N}\right)\right)}
$$

which is a probability measure supported in $[-\|z\|,\|z\|]$.

It is not difficult to show (see Sect. 4 and [LPS], as well as [Sa1] and [Ser] for analogous equidistribution results) that there are measures $v^{\text {even }}(z)$ and $v^{\text {odd }}(z)$ which are determined entirely by the abstract group $\Gamma_{z}$ and its generators (that is $\operatorname{supp}(z)$ ), such that $\mu_{2 N}(z) \rightarrow v^{\text {even }}(z)$ and $\mu_{2 N+1}(z) \rightarrow v^{\text {odd }}(z)$, as $N \rightarrow \infty$. We call $v^{\text {even }}(z)$ and $v^{\text {odd }}(z)$ the densities of states. The finer question as to how this equidistribution takes place depends on $\operatorname{supp}(z)$ and how it sits in $G$. We say that $g_{1}, \ldots, g_{k} \in G$ are diophantine if there is a $B=B\left(g_{1}, \ldots, g_{k}\right)>0$ such that for $m \geq 1$ and $R_{m}$ a word in $g_{1}, \ldots, g_{k}$ of length $m$ and $R_{m} \neq \pm e$, we have

$$
\left\|R_{m} \pm e\right\| \geq B^{-m}
$$

Here

$$
\left\|\left[\begin{array}{ll}
a & b \\
c & d
\end{array}\right]\right\|^{2}=|a|^{2}+|b|^{2}+|c|^{2}+|d|^{2} .
$$

It is not hard to show (see Proposition 4.3) that if $g_{1}, \ldots, g_{k} \in M_{2}(\overline{\mathbf{Q}})$, that is to say they have algebraic number entries, then $g_{1}, \ldots, g_{k}$ are diophantine. On the other hand, the topologically generic (i.e. in the sense of Baire category) $g_{1}, \ldots, g_{k} \in G^{(k)}$ is not diophantine.

Theorem 1.3. Assume that $\operatorname{supp}(z)$ is diophantine and that $v^{\text {even }}(z)=$ $f^{(\text {even })}(x) d x, v^{\text {odd }}(z)=f^{(\text {odd })}(x) d x$ with $f^{(\text {even })}, f^{(\text {odd })}$ in $L^{\infty}(\mathbf{R})$, then for $N$ large

$$
D\left(\mu_{2 N}(z), v^{\text {even }}(z)\right) \ll_{z} \frac{1}{\log N}
$$


and

$$
D\left(\mu_{2 N+1}(z), v^{o d d}(z)\right) \ll_{z} \frac{1}{\log N}
$$

(here $D(v, \mu)$ is the discrepancy between the measures $v$ and $\mu$, that is $D(\nu, \mu)=\sup \{|v(I)-\mu(I)|: I=[a, b] \subset \mathbf{R}\})$.

Theorem 1.3 is false for the topologically generic $z=g_{1}+g_{1}^{-1}+\ldots+$ $g_{k}+g_{k}^{-1},\left(g_{1}, \ldots, g_{k}\right) \in G^{(k)}$. On the other hand the numerical results of Sect. 5 suggest that for $k \geq 2$ fixed and $z_{g}$ chosen with $g \in G^{(k)}$, generic in the measure sense, the discrepancy should behave like those for random matrix models, that is it should be $O((\log N) / N)$, see Fig. 1 which displays $\mu_{N}(z)$ against $\nu(z)$ for such a random $z$. So for such $z$ 's the result in Theorem 1.3 is probably very far from the truth. For the Ramanujan element $z_{q}$ (defined in Remark 1) the discrepancy is not small, see Fig. 2 for a similar comparison. Note that it has a very large centralizer in $\mathbf{R}[G]$, in fact the $z_{q}$ 's all commute with each other. So the eigenvalues of $\widehat{z}_{q}\left(\pi_{N}\right)$ should not be expected to behave like any random matrix ensemble. In fact experience with numerics for the spectra of Laplacians for arithmetic hyperbolic surfaces [BGGS,BSS] suggest that $\operatorname{spec}\left(\widehat{z}_{q}\left(\pi_{N}\right)\right)$ is more likely to behave like random numbers (i.e. "Poisson"). The following lower bound confirms this and is the analogue of the lower bounds for the remainder term in Weyl's law for arithmetic hyperbolic surfaces, see [Hej] and [LS].

Theorem 1.4. Fix $q \geq 3$ and $z_{q}$ as above, a Ramanujan element. There is a sequence $N_{j} \rightarrow \infty$ such that

$$
D\left(\mu_{\pi_{N_{j}}}\left(z_{S_{p}}\right), v(z)\right) \gg \frac{1}{N_{j}^{1 / 2}\left(\log N_{j}\right)^{2}}
$$

In Sect. 5 we report on numerical experiments concerning the "unfolded" spacing distribution between the eigenvalues (5) as $N$ gets large. Consider the generic in measure $z=z_{g}=g_{1}+g_{1}^{-1}+\ldots+g_{k}+g_{k}^{-1}$, $g=\left(g_{1}, \ldots, g_{k}\right) \in G^{(k)}$. For $k=2$ the centralizer of $z \in \mathbf{R}[G]$ consists of more than just the polynomial ring in $z, \mathbf{R}[z]$. Indeed by conjugating such an element by a suitable member of $G$ we can assume that

$$
g_{1}=\left[\begin{array}{ll}
\lambda & 0 \\
0 & \bar{\lambda}
\end{array}\right], \quad g_{2}=\left[\begin{array}{cc}
a_{1} & b_{1} \\
-\overline{b_{1}} & \overline{a_{1}}
\end{array}\right] .
$$

Put $c=\left(-b_{1} / \overline{b_{1}}\right)^{1 / 2}$. Then

$$
\left[\begin{array}{cc}
0 & c \\
-\bar{c} & 0
\end{array}\right]
$$

commutes with $z$. That is for $k=2$ there is a persistent symmetry of order 2 (note that this symmetry is not broken by passing to $z=x\left(g_{1}+g_{1}^{-1}\right)+$ 
$y\left(g_{2}+g_{2}^{-1}\right)$ with $\left.x, y \in \mathbf{R}\right)$. However for $k \geq 3$ and $g=\left(g_{1}, \ldots, g_{k}\right)$ generic it appears that the centralizer of $z$ in $\mathbf{R}[G]$ is no bigger than $\mathbf{R}[z]$. In particular the generic such $z$ is "desymmetrized" and we examine the consecutive spacing distribution between the eigenvalues of $\widehat{z}\left(\pi_{N}\right)$. For such a random $z$ with $k=3$ we found universally that these spacings followed the GOE spacing law for $N$ going to infinity through even values and GSE spacing laws for $N$ odd. Figs. 3 and 4 give typical examples of this phenomenon. On the other hand the same question for the Ramanujan element $z_{5}$ yielded a Poissonian consecutive spacing distribution (after the obvious symmetries have been taken into account) - see Figs. 5 and 6 in Sect. 5. The element $\tilde{z}_{7}$ (see the example after Theorem 1.1) is special in that it is formed out of integral quaternion matrices though $\Gamma_{\tilde{z}_{7}}$ is not arithmetic. We found that its spacings (again after a symmetry analysis) are GOE and GSE depending on the parity. A typical example of this data is given in Fig. 7. For details about the data and computations see Sect. 5 .

Note that for the random $g \in G^{(k)}, \Gamma=\left\langle g_{1}, \ldots, g_{k}\right\rangle$ acts ergodically on $S^{2}$. These results point to the conjecture that for $k \geq 3$ fixed and $g \in G^{(k)}$ given and generic (in measure) the eigenvalues of the quantization $\widehat{z}_{g}\left(\pi_{N}\right)$ follow GOE spacing statistics as $N$ goes to infinity through even values, while they follow GSE statistics as $N$ goes to infinity through odd values. Besides this conjecture (which if true is no doubt difficult to prove) there are a number of more tractable problems that remain. We mention some of these:

(1) Prove that for $k \geq 2$ and $g \in G^{(k)}$ generic in measure, $z_{g}=g_{1}+g_{1}^{-1}+$ $\ldots+g_{k}+g_{k}^{-1}$ has a gap (see [LPS]).

(2) With our present knowledge it is conceivable that $z_{g}$ has a gap as long as $\Gamma_{z_{g}}$ acts ergodically on $S^{2}$ (cf. [LW]).

(3) Consider the parameter space ( $k \geq 2$, fixed) $G^{(k)} / \sim$, where $z_{g}$ is as above and $z_{g} \sim z_{\tilde{g}}$ if $\delta\left(g_{1}, \ldots, g_{k}\right) \delta^{-1}=\left(\tilde{g}_{1}, \ldots, \tilde{g}_{k}\right)$ with $\delta \in G$. What can be said about the Ramanujan elements $z \in G^{(k)} / \sim$ ? Is this set finite or infinite? One can show it is closed and nowhere dense (cf. [LPS, Theorem 1.4]; in fact its intersection with any 1-dimensional curve is closed and nowhere dense in that curve).

(4) Prove that the generic $\left(g_{1}, \ldots, g_{k}\right) \in G^{(k)}$ in the sense of measure is diophantine.

(5) One can ask similar questions for $\mathbf{R}[G]$ where $G$ is a more general compact topological group or for $\mathbf{R}[\Gamma]$ relative to a family $\pi_{N}$ of unitary finite dimensional representations of $\Gamma$ arising from geometry or quantization. 


\section{Properties of $\pi_{N}$}

With notation as in Sect. 1 let $e_{0}, e_{1}, \ldots e_{N}$ be the basis of $W_{N+1}$ given by

$$
e_{j}=x^{j} y^{N-j}, \quad j=0,1, \ldots, N .
$$

The bilinear form $\langle$,$\rangle on W_{N+1}$ defined by

$$
\left\langle e_{j}, e_{k}\right\rangle=\left(\begin{array}{c}
N \\
j
\end{array}\right)^{-1}(-1)^{j} \delta_{j, N-k}
$$

is nondegenerate and is preserved by $\pi_{N}$. That is $\left\langle\pi_{N}(g) v, \pi_{N}(g) w\right\rangle=$ $\langle v, w\rangle$ for $g \in G$ and $v, w \in W_{N+1}$. The form is symmetric for $N$ even and skew-symmetric for $N$ odd. It follows that $\pi_{N}$ is orthogonal for $N$ even and symplectic for $N$ odd. Hence with a suitable basis (over $\mathbf{C}$ ) we can assume that that $\pi_{N}$ preserves

$$
I_{N+1}=\left[\begin{array}{cccc}
1 & 0 & \ldots & 0 \\
0 & 1 & \ldots & 0 \\
\vdots & \vdots & \ddots & \vdots \\
0 & 0 & \ldots & 1
\end{array}\right]
$$

for $N$ even and

$$
J=\left[\begin{array}{cccc}
E & 0 & \ldots & 0 \\
0 & E & \ldots & 0 \\
\vdots & \vdots & \ddots & \vdots \\
0 & 0 & \ldots & E
\end{array}\right], \quad E=\left[\begin{array}{cc}
0 & 1 \\
-1 & 0
\end{array}\right]
$$

for $N$ odd. That is

$$
\begin{cases}\left(\pi_{N}(g)\right)^{t} \pi_{N}(g)=I_{N+1}, & N \text { even } ; \\ \left(\pi_{N}(g)\right)^{t} J \pi_{N}(g)=J, & N \text { odd } .\end{cases}
$$

Furthermore for $g \in \mathrm{SU}(2)$ we can arrange for $\pi_{N}(g)$ to be unitary with respect to the standard Hermitian form on $W_{N+1}$. Thus for $N$ even $\pi_{N}(g) \in \mathrm{O}(N+1, \mathbf{R})$ while for $N$ odd $\pi_{N}(g) \in \mathrm{USp}(N+1)$.

If $N$ is even $\left(\pi_{N}(g)+\pi_{N}\left(g^{-1}\right)\right)^{t}=\pi_{N}(g)+\pi_{N}\left(g^{-1}\right)$. It is also selfadjoint hence $\pi_{N}(g)+\pi_{N}\left(g^{-1}\right)$ is a real symmetric matrix. It follows that for any $z \in \mathbf{R}[G]$ which is selfadjoint, $\widehat{z}\left(\pi_{N}\right)$ lies in the real linear space $\mathcal{H}_{N+1}$ of $(N+1) \times(N+1)$ real symmetric matrices.

For $N$ odd, $J^{t}\left(\pi_{N}(g)+\pi_{N}\left(g^{-1}\right)\right) J=\left(\pi_{N}(g)+\pi_{N}\left(g^{-1}\right)\right)^{t}$. Also $\pi_{N}(g)+$ $\pi_{N}\left(g^{-1}\right)$ is selfadjoint so we see that $\widehat{z}\left(\pi_{N}\right)$ lies in the real linear space $\mathcal{H}_{N+1}$ of $(N+1) \times(N+1)$ complex matrices satisfying

$$
J^{t} H J=H^{t} \text { and } H^{*}=H .
$$


It is easily seen that any $H \in \mathcal{H}_{N+1},(N+1=2 M)$ has its eigenvalues consist of $M$ eigenvalues each of multiplicity 2 (of course some of these $M$ eigenvalues may coincide).

For the generic $\left(g_{1}, g_{2}, \ldots, g_{k}\right) \in G^{k}$ (by which we mean here outside a countable union of codimension 1 subsets $), k \geq 2, z\left(g_{1}, g_{2}, \ldots, g_{k}\right)=$ $g_{1}+g_{1}^{-1}+g_{2}+g_{2}^{-1}+\ldots+g_{k}+g_{k}^{-1}$ has the property that $\widehat{z}\left(\pi_{N}\right)$ has simple spectrum if $N$ is even and has $M=(N+1) / 2$ distinct eigenvalues each of multiplicity 2 if $N$ is odd. To see this it suffices from general principles involving the discriminant of a matrix, to exhibit one such $\left(g_{1}, g_{2}, \ldots, g_{k}\right)$. For $\alpha \in \mathbf{R}$ and e $(\alpha)=e^{2 \pi i \alpha}$ let $z(\alpha) \in \mathbf{R}[G]$ be given by

$$
z(\alpha)=\left[\begin{array}{cc}
\mathrm{e}(\alpha) & 0 \\
0 & \mathrm{e}(-\alpha)
\end{array}\right]+\left[\begin{array}{cc}
\mathrm{e}(-\alpha) & 0 \\
0 & \mathrm{e}(\alpha)
\end{array}\right]+(k-1)\left(\left[\begin{array}{cc}
0 & 1 \\
-1 & 0
\end{array}\right]+\left[\begin{array}{cc}
0 & -1 \\
1 & 0
\end{array}\right]\right) .
$$

A straightforward calculation yields

$$
\begin{array}{r}
\operatorname{spec}\left(\widehat{z(\alpha)}\left(\pi_{N}\right)\right)=\{2 \cos N \alpha \pm(2 k-2), \\
\left.2 \cos (N-2) \alpha \pm(2 k-2), \ldots, 2+(-1)^{N / 2}(2 k-2)\right\}
\end{array}
$$

if $N$ is even and

$$
\begin{array}{r}
\operatorname{spec}\left(\widehat{z(\alpha)}\left(\pi_{N}\right)\right)=\{2 \cos N \alpha, 2 \cos N \alpha, \\
2 \cos (N-2) \alpha, 2 \cos (N-2) \alpha, \ldots, 2 \cos \alpha, 2 \cos \alpha\}
\end{array}
$$

if $N$ is odd.

Hence if we choose $\alpha$ generically (e.g. take e $(\alpha)$ to be transcendental) then $z(\alpha)$ will satisfy the claimed property. Note that this $z(\alpha)$ fails to have a gap. The support group $\Gamma_{z(\alpha)}$ is a dihedral group and the densities of states $v^{\text {even }}(z)$ and $v^{\text {odd }}(z)$ are easily determined from the explicit description of the spectrum: $v^{\text {odd }}(z(\alpha))=d y / \sqrt{4-y^{2}}$ on $[-2,2]$ while $v^{\text {even }}(z(\alpha))$ is half of this measure translated by $\pm 2(k-1)$.

While our interest lies in $\operatorname{spec}\left(\widehat{z}\left(\pi_{N}\right)\right)$ as $N \rightarrow \infty$ for $z$ fixed, we note that if we let $k$ go to infinity as well then the spectrum of the generic $\widehat{z}\left(g_{1}, g_{2}, \ldots, g_{k}\right)\left(\pi_{N}\right)$ will follow the GOE laws (see [M] for definitions) if $N$ is even and the GSE laws if $N$ is odd.

Proposition 2.1. Let $v_{N, k}$ be the direct image of $d g_{1} d g_{2} \ldots d g_{k}$ on $G^{(k)}$ under the map

$$
\left(g_{1}, g_{2}, \ldots, g_{k}\right) \rightarrow\left(\frac{1}{\sqrt{k}}\left(g_{1}+g_{1}^{-1}+\ldots+g_{k}+g_{k}^{-1}\right)\right)^{-}\left(\pi_{N}\right)
$$

Thus $v_{N, k}$ is a probability measure on $\mathcal{H}_{N+1}$. As $k \rightarrow \infty, v_{N, k}$ converges in measure to the standard GOE measure on $\mathcal{H}_{N+1}$ if $N$ is even and to the standard GSE measure if $N$ is odd. 
Proof. The measure $v_{N, k}$ on the real vector space $\mathcal{H}_{N+1}$ is a sum of i.i.d. random variables. We may therefore appeal to the general vector valued central limit theorem. The distribution of the individual summand is $H(g)=$ $\pi_{N}(g)+\pi_{N}\left(g^{-1}\right), g \in G$. Thus once we show that $\int_{G} H(g) d g=0$ we know that the limit of $v_{N, k}$ as $k \rightarrow \infty$ is a Gaussian. The issue is to identify this Gaussian and where it is supported.

We begin with the case that $N$ is even. $H(g)=\left(h_{i j}(g)\right)$ is real symmetric so we can consider the $(N+1)(N+2) / 2$ dimensional space $h_{i j}, 1 \leq i \leq$ $j \leq N+1$, i.e. $\mathcal{H}_{N+1}$. We assert that

$$
\int_{G} h_{i j}(g) d g=0
$$

for $1 \leq i \leq j \leq N+1$ and

$$
\int_{G} h_{i j}(g) h_{r s}(g) d g=\frac{2}{N+1}\left(\delta_{i s} \delta_{j r}+\delta_{i r} \delta_{j s}\right)
$$

To see this recall that since $\pi_{N}$ is irreducible we have from Schur's Lemma [H] (assuming $N \geq 1$ ) that

$$
\int_{G} 1 \cdot \pi_{N}(i, j)(g) d g=0
$$

and

$$
\int_{G} \pi_{N}(i, j)(g) \pi_{N}(m, n)\left(g^{-1}\right) d g=\frac{\delta_{i n} \delta_{j m}}{N+1}
$$

for any $1 \leq i, j, m, n \leq N+1$.

Thus (15) follows from (17) while (16) from (18) together with the fact that $\pi_{N}(g)$ is orthogonal and hence

$$
\int_{G} \pi_{N}(i, j)(g) \pi_{N}(r, s)(g) d g=\int_{G} \pi_{N}(i, j)(g) \pi_{N}(s, r)\left(g^{-1}\right) d g=\frac{\delta_{i r} \delta_{j s}}{N+1} .
$$

The equalities (15) and (16) identify the covariance-matrix for the limiting Gaussian as being:

$$
\begin{gathered}
C_{N} e^{-\frac{N+1}{4}\left(h_{11}^{2}+\ldots+h_{N+1, N+1}^{2}\right)-\frac{N+1}{2}\left(\sum_{1 \leq i<j \leq N+1} h_{i j}^{2}\right)} \prod_{1 \leq i \leq j \leq N+1} d h_{i j}= \\
C_{N} e^{-\frac{N+1}{4} \operatorname{tr}\left(H^{2}\right)} \prod_{i \leq j} d h_{i j}
\end{gathered}
$$

This Gaussian is $\mathrm{O}(N+1)$ invariant on $\mathcal{H}_{N+1}$ and is exactly the GOE measure on $\mathcal{H}_{N+1}$, see [M, p. 39]. 
The case that $N$ is odd is a little more complicated. The linear space over $\mathbf{R}$ of $(N+1) \times(N+1)=2 M \times 2 M$ matrices $H$ satisfying (14) takes the form

$$
\begin{gathered}
H=\left[\begin{array}{ccccccc}
x_{11} & 0 & z_{12} & w_{12} & \ldots & z_{1 M} & w_{1 M} \\
0 & x_{11} & -\overline{w_{12}} & \overline{z_{12}} & \ldots & -\overline{w_{1 M}} & \overline{z_{1 M}} \\
& x_{22} & 0 & & & \\
\vdots & 0 & x_{22} & & \\
z_{M 1} & w_{M 1} & & & & \\
-\overline{w_{M 1}} & \frac{}{z_{M 1}} & & \ldots & 0 & x_{M M}
\end{array}\right] \\
=\left[\begin{array}{cccc}
A_{11} & A_{12} & \ldots & A_{1 M} \\
A_{21} & A_{22} & \ldots & A_{2 M} \\
\vdots & & \ddots & \vdots \\
A_{M 1} & A_{M 2} & \ldots & A_{M M}
\end{array}\right]
\end{gathered}
$$

where

$$
A_{j j}=\left[\begin{array}{cc}
x_{j j} & 0 \\
0 & x_{j j}
\end{array}\right], \quad x_{j j} \in \mathbf{R}
$$

for $1 \leq j \leq M$ and

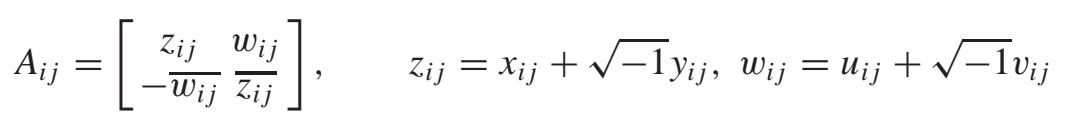

for $1 \leq i<j \leq M$.

Hence $\mathcal{H}_{N+1}$ is spanned over the reals by

$$
x_{j j}, \quad j=1, \ldots, M
$$

and

$$
x_{i j}, y_{i j}, u_{i j}, v_{i j}, \quad 1 \leq i<j \leq M .
$$

It is therefore of dimension $M(2 M-1)$. Again $v_{N, k}$ is a sum of i.i.d. random variables in $\mathcal{H}_{N+1}$, the individual summand being $H(g)=\pi_{N}(g)+$ $\pi_{N}\left(g^{-1}\right), g \in G$ as before. The equalities (17) and (18) continue to hold since $\pi_{N}$ is irreducible so $\int_{G} H(g) d g=0$. Thus $v_{N, k}$ converges to a Gaussian on $\mathcal{H}_{N+1}$ and we need to identify the covariance matrix. The relation (13) yields

$$
\left(\pi_{N}(g)\right)^{t}=\left[\begin{array}{ccccc}
a_{11}(g) & b_{11}(g) & \ldots & a_{1 M}(g) & b_{1 M}(g) \\
c_{11}(g) & d_{11}(g) & \ldots & c_{1 M}(g) & d_{1 M}(g) \\
\vdots & & \ddots & \\
a_{M 1}(g) & b_{M 1}(g) & \ldots & a_{M M}(g) & b_{M M}(g) \\
c_{M 1}(g) & d_{M 1}(g) & \ldots & c_{M M}(g) & d_{M M}(g)
\end{array}\right]^{t}
$$




$$
=\left[\begin{array}{ccccc}
d_{11}\left(g^{-1}\right) & -c_{11}\left(g^{-1}\right) & \ldots & d_{1 M}\left(g^{-1}\right) & -c_{1 M}\left(g^{-1}\right) \\
-b_{11}\left(g^{-1}\right) & a_{11}\left(g^{-1}\right) & \ldots & -b_{1 M}\left(g^{-1}\right) & a_{1 M}\left(g^{-1}\right) \\
\vdots & & \ddots & & \vdots \\
d_{M 1}\left(g^{-1}\right) & -c_{M 1}\left(g^{-1}\right) & \ldots & d_{M M}\left(g^{-1}\right) & -c_{M M}\left(g^{-1}\right) \\
-b_{M 1}\left(g^{-1}\right) & a_{M 1}\left(g^{-1}\right) & \ldots & -b_{M M}\left(g^{-1}\right) & a_{M M}\left(g^{-1}\right)
\end{array}\right] .
$$

From (19) one sees easily that the covariance matrix of $H(g) \in \mathcal{H}_{N+1}$ is diagonal. Its diagonal entries may be computed using (17), (18) and (19):

$$
\begin{aligned}
\int_{G} x_{j j}^{2}(g) d g & =\int_{G}\left(a_{j j}(g)+a_{j j}\left(g^{-1}\right)\right)\left(a_{j j}(g)+a_{j j}\left(g^{-1}\right)\right) d g \\
& =\frac{2}{N+1}+2 \int_{G} a_{j j}^{2}(g) d g \\
& =\frac{2}{N+1}+2 \int_{G} a_{j j}(g) d_{j j}\left(g^{-1}\right) d g \\
& =\frac{2}{N+1} .
\end{aligned}
$$

Similarly

$$
\begin{aligned}
\int_{G} z_{i j}(g) \overline{z_{i j}}(g) d g & =\frac{2}{N+1}, \\
\int_{G} z_{i j}^{2}(g) d g & =\int_{G} \bar{z}_{i j}^{2}(g) d g=0 . \\
\int_{G} w_{i j}(g) \overline{w_{i j}}(g) d g & =\frac{2}{N+1}, \\
\int_{G} w_{i j}^{2}(g) d g & =0 .
\end{aligned}
$$

Hence for $1 \leq i<j \leq M$,

$$
\int_{G} x_{i j}^{2}(g) d g=\int_{G} y_{i j}^{2}(g) d g=\int_{G} u_{i j}^{2}(g) d g=\int_{G} v_{i j}^{2}(g) d g=\frac{1}{N+1}
$$

while

$$
\int_{G} x_{i j}(g) y_{i j}(g) d g=0
$$

etc.

Thus the Gaussian limit takes the form

$$
\begin{gathered}
C_{N} e^{-\frac{N+1}{2}\left(\sum_{j=1}^{M} x_{j j}^{2}\right)-(N+1)\left(\sum_{1 \leq i<j \leq M}\left(x_{i j}^{2}+y_{i j}^{2}+u_{i j}^{2}+v_{i j}^{2}\right)\right)} \\
\times \prod_{j=1}^{M} d x_{j j} \prod_{1 \leq i<j \leq M} d x_{i j} d y_{i j} d u_{i j} d v_{i j}=
\end{gathered}
$$




$$
C_{N} e^{-\frac{N+1}{4} \operatorname{tr}\left(H^{2}\right)} \prod_{j=1}^{M} d x_{j j} \prod_{1 \leq i<j \leq M} d x_{i j} d y_{i j} d u_{i j} d v_{i j} .
$$

This is precisely the $\operatorname{USp}(2 M)$ invariant Gaussian on $\mathcal{H}_{2 M}$, that is the GSE measure, see [M, p. 41].

\section{The gap}

In what follows we exhibit $z$ 's with a gap. We take $z$ 's in $\mathbf{R}[G]$ of the form

$$
z=g_{1}+g_{1}^{-1}+g_{2}+g_{2}^{-1}+\ldots+g_{k}+g_{k}^{-1}
$$

Assume further that $\left\langle g_{1}, g_{2}, \ldots, g_{k}\right\rangle=\Gamma_{z}$ is a free group on these generators and that $k \geq 2$. Hausdorff [Hau] in his work on what later became known as the "Hausdorff-Banach-Tarski" paradox [Wa], exhibits such $g$ 's. In fact it is easy to see that the generic $\left(g_{1}, g_{2}, \ldots, g_{k}\right) \in G^{(k)}$ (in the sense of being outside a countable union of co-dimension one sets) gives a free $\Gamma_{z}$. Now such a group $(k \geq 2)$ being free is Zariski dense in $\mathrm{SL}_{2}(\mathbf{C})$. The representations $\pi_{N}$ extend to $\mathrm{SL}_{2}(\mathbf{C})$ and are irreducible. Hence if $v \in W_{N+1}$ satisfies $\pi_{N}(\Gamma) v=\lambda v$ for some $|\lambda|=1$ then $\pi_{N}\left(\mathrm{SL}_{2}\right) v=\lambda v$ and hence $v=0$. It follows that $2 k$ and $-2 k$ are not in $\operatorname{spec}\left(\widehat{z}\left(\pi_{N}\right)\right)$, for if $\widehat{z}\left(\pi_{N}\right) v= \pm 2 k v$ then $\pi_{N}\left(g_{j}\right) v= \pm v$ for $1 \leq j \leq k$ and hence $\pi_{N}(\Gamma) v= \pm v$ and $v=0$. Thus for any $z$ with $\Gamma_{z}$ free, $\operatorname{spec}\left(\widehat{z}\left(\pi_{N}\right)\right) \subset(-2 k, 2 k)$. In particular for such a $z$ for which we can establish a gap (for $N$ large) we will have

$$
\sup _{N \geq 1}\left\|\widehat{z}\left(\pi_{N}\right)\right\|<\|z\|_{1}=2 k
$$

Let $p=2 k-1$ (we do not assume that $p$ is prime) and let $U_{n}(\cos \theta)=$ $\sin (n+1) \theta / \sin \theta$ for $n \geq 0$ be the $n$-th Chebyshev polynomial of the second kind. In $\mathbf{R}[G]$ we have the following relation which is easily established inductively [LPS]

$$
p^{n / 2} U_{n}(z /(2 \sqrt{p}))=\sum_{|\omega| \leq n}^{\prime} \omega
$$

where the sum is over all reduced words $\omega$ in $g_{1}, g_{1}^{-1}, g_{2}, g_{2}^{-1}, \ldots g_{k}, g_{k}^{-1}$ of length $|\omega|=m \leq n$ with $m \equiv n(\bmod 2)$. The image of this relation under $\pi_{N}$ yields

$$
p^{n / 2} U_{n}\left(\widehat{z}\left(\pi_{N}\right) /(2 \sqrt{p})\right)=\sum_{|\omega| \leq n}^{\prime} \widehat{\omega}\left(\pi_{N}\right) .
$$


Write the eigenvalues $\lambda_{j}$ of $\widehat{z}\left(\pi_{N}\right)$ as

$$
\lambda_{j}=2 \sqrt{p} \cos \left(\theta_{j, N}\right), \quad j=0,1, \ldots, N
$$

where

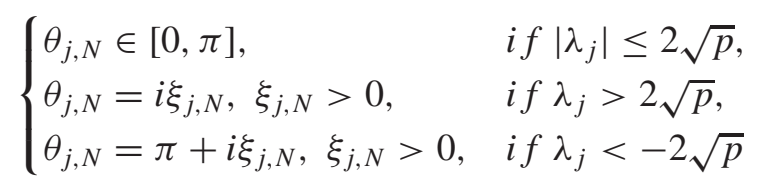

We call the $\theta_{j, N}$ 's not in $[0, \pi]$ exceptional. Indeed since $\Gamma_{z}$ is free most of the $\theta$ 's are not exceptional as $N \rightarrow \infty$ (cf. Sect. 4).

Each $g \in G$ is conjugate to a diagonal matrix

$$
\left[\begin{array}{cc}
\mathrm{e}\left(r_{g}\right) & 0 \\
0 & \mathrm{e}\left(-r_{g}\right)
\end{array}\right]
$$

with $0 \leq r_{g} \leq \pi$ which is determined from

$$
\operatorname{tr}(g)=2 \cos r_{g}
$$

Taking the trace of both sides of (23) yields

$$
p^{n / 2} \sum_{j=0}^{N} \frac{\sin (n+1) \theta_{j, N}}{\sin \theta_{j, N}}=\sum_{|\omega| \leq n} \frac{\sin (N+1) r_{\omega}}{\sin r_{\omega}} .
$$

We use (27) to estimate the number of exceptional $\theta_{j, N}$ 's. To this end note that if $n$ is even (which we assume henceforth in this section) then for exceptional $\theta_{j, N}$ we have

$$
\frac{\sin (n+1) \theta_{j, N}}{\sin \theta_{j, N}}=\frac{\sinh (n+1) \xi_{j, N}}{\sinh \xi_{j, N}}>0 .
$$

Hence (27) becomes

$$
\sum_{\substack{\xi_{j, N} \\ \text { exceptional }}} \frac{\sinh (n+1) \xi_{j, N}}{\sinh \xi_{j, N}}+O(N n)=p^{-n / 2} \sum_{0<|\omega| \leq n} \frac{\sin (N+1) r_{\omega}}{\sin r_{\omega}}
$$

In order to exploit the cancellation in the sum on the right hand side of (29) we sum over $N$ in a range $N \sim N_{0}, N_{0}$ large. For technical reasons we do so in a smooth way. Let $f \in C^{\infty}(1 / 2,3 / 2), f \geq 0$ and $f(1)=1$. Summing over $N$ in (29) we get

$$
\sum_{N \sim N_{0}} f\left(\frac{N+1}{N_{0}+1}\right) \sum_{\substack{\xi_{j, N} \\ \text { exceptional }}} \frac{\sinh (n+1) \xi_{j, N}}{\sinh \xi_{j, N}}=
$$




$$
=p^{-n / 2} \sum_{0<|\omega| \leq n} \sum_{N \sim N_{0}} f\left(\frac{N+1}{N_{0}+1}\right) \frac{\sin (N+1) r_{\omega}}{\sin r_{\omega}}+O\left(N_{0}^{2} n\right)
$$

Poisson summation applied to the sum on $N$ leads to the following: For any $\delta>0, A \gg 1$ and $r \in[0, \pi]$ we have

$$
\begin{gathered}
\sum_{N \sim N_{0}} f\left(\frac{N+1}{N_{0}+1}\right) \frac{\sin (N+1) r}{\sin r} \ll_{\delta, A} \\
\begin{cases}N_{0}^{2}, & r \in\left[0, N_{0}^{-1+\delta}\right], \\
\min \left\{N_{0}^{2}, \frac{N_{0}^{-A}}{\sin r}\right\}, & r \in\left[N_{0}^{-1+\delta}, \pi\right] .\end{cases}
\end{gathered}
$$

Since $\Gamma_{z}$ is free we have $0<r_{\omega}<\pi$ for any reduced word $\omega(|\omega|>0)$. In fact we assume that there is a constant $B=B\left(g_{1}, \ldots, g_{k}\right)$ such that for $|\omega|=n$,

$$
B^{-n} \leq r_{\omega} \leq \pi-B^{-n}
$$

We will verify (32) for any of the choices of $g_{1}, \ldots, g_{k}$ below. Since $A$ is arbitrarily large in (31), we conclude from (30) and (31) that

$$
\begin{gathered}
\sum_{N \sim N_{0}} f\left(\frac{N+1}{N_{0}+1}\right) \sum_{\substack{\xi_{j, N} \\
\text { exceptional }}} \frac{\sinh (n+1) \xi_{j, N}}{\sinh \xi_{j, N}} \ll_{\delta, A} \\
N_{0}^{2} n+p^{-n / 2} N_{0}^{2} \#\left\{0<|\omega|<n: r_{\omega} \in\left[0, N_{0}^{-1+\delta}\right]\right\} .
\end{gathered}
$$

Thus estimating the left hand side above is reduced to estimating the number fo words $\omega^{\prime},\left|\omega^{\prime}\right|<n$ with small rotation (i.e. size $N_{0}^{-1+\delta}$ ). Before continuing we contemplate what to expect as far as the number of such $r_{\omega}$ 's. Going back to (27) we note that for $N$ fixed if we let $n \rightarrow \infty$ and use the fact that $-2 k<\lambda_{j, N}<2 k$ for $N \geq 1$, we get

$$
\lim _{n \rightarrow \infty} p^{-n} \sum_{|\omega| \leq n} \frac{\sin (N+1) r_{\omega}}{\sin r_{\omega}}=0 .
$$

The number of $|\omega| \leq n$ is $(p+1) p^{n-1}$ so (34) asserts that the rotations $r_{\omega},|\omega| \leq n$ become equidistributed with respect to $2 \sin ^{2} \theta d \theta / \pi$, that is with respect to the Weyl measure which is the image of the Haar measure on $G$ onto the maximal torus. We might expect at least generically for $\left(g_{1}, \ldots, g_{k}\right) \in G^{(k)}$ that this continues to hold approximately for small intervals. That is that

$$
\#\left\{|\omega| \leq n \mid r_{\omega} \in\left[0, N_{0}^{-1+\delta}\right]\right\} \ll \frac{\{\omega:|\omega| \leq n\}}{N_{0}^{3-3 \delta}}+1 .
$$


While we don't know how to establish (35) for the generic $\left(g_{1}, \ldots, g_{k}\right)$, we can prove it for some special choices.

Granting (35) we have (using (28) and (33)) that

$$
\sum_{\substack{\xi_{j, N N_{0}} \\ \text { exceptional }}} \frac{\sinh (n+1) \xi_{j, N_{0}}}{\sinh \xi_{j, N_{0}}} \ll N_{0}^{2} n+p^{-n / 2}\left(\frac{p^{n}}{N_{0}^{3}}+1\right) N_{0}^{2}
$$

and hence for $b>0$

$$
\sum_{e^{\xi j, N_{0}} \geq p^{b}} 1 \ll p^{-n b}\left(N_{0}^{2} n+p^{-n / 2} N_{0}^{2}\left(\frac{p^{n}}{N_{0}^{3}}+1\right)\right)
$$

Choose $n$ satisfying $N_{0}^{6} \leq p^{n} \leq p^{2} N_{0}^{6}$ ( $n$ even), then

$$
\sum_{e^{\xi_{j, N}} N_{0} \geq p^{b}} 1 \ll p^{(-b+1 / 3) n} \cdot n
$$

If $b>1 / 3$ the right hand side of (36) is less than one and we conclude that for $N_{0}$ large there are no exceptional $\xi_{j, N_{0}}$ with $e^{\xi_{j, N_{0}}} \geq p^{b}$. That is for $N_{0}$ large and any $j$

$$
\left|\lambda_{j, N_{0}}\right| \leq p^{1 / 2}\left(p^{1 / 3}+p^{-1 / 3}\right)+\tilde{\varepsilon},
$$

( $\tilde{\varepsilon}$ sufficiently small).

So the explicit gap (36) is what we expect to hold for generic $\left(g_{1}, \ldots, g_{k}\right)$ and we now exhibit $g$ 's satisfying (35) (or sufficiently good approximations to it).

Let $\mathbf{H}(\mathbf{Z})$ be the integral Hamilton quaternions, so if $\alpha \in \mathbf{H}(\mathbf{Z})$ then $\alpha=x_{0}+x_{1} \mathrm{i}+x_{2} \mathrm{j}+x_{3} \mathrm{k}$ with $x_{j} \in \mathbf{Z}$. We let $\bar{\alpha}=x_{0}-x_{1} \mathrm{i}-x_{2} \mathrm{j}-x_{3} \mathrm{k}$ and $N(\alpha)=\bar{\alpha} \alpha \in \mathbf{Z}$. For $q \geq 3$ a prime number we take for $\tilde{g}_{1}, \tilde{g}_{2}, \ldots \tilde{g}_{k}$ a subset of the set $\{\alpha \mid N(\alpha)=q\}$ (as pointed out in the Introduction this set has $8(q+1)$ elements $)$ which satisfies

(I) $\tilde{g}_{j_{1}} \neq \epsilon \tilde{g}_{j_{2}}$ if $j_{1} \neq j_{2}$ and $\epsilon \in\{ \pm 1, \pm \mathrm{i}, \pm \mathrm{j}, \pm \mathrm{k}\}$ is a unit.

(II) $\tilde{g}_{j_{1}} \neq \epsilon \overline{\tilde{g}_{j_{2}}}$ for any $j_{1}, j_{2}$ and $\epsilon$ a unit.

As is well known (see [LPS] for example) different reduced words $\omega=$ $R\left(\tilde{g}_{1}, \overline{\tilde{g}_{1}}, \ldots \overline{\tilde{g}_{k}}\right)$ in $\tilde{g}_{1}, \ldots, \overline{\tilde{g}_{k}}$ of length $m \geq 1$ give different quaternions of norm $q^{m}$. In particular to each such word corresponds a unique solution of

$$
x_{0}^{2}+x_{1}^{2}+x_{2}^{2}+x_{3}^{2}=q^{m}, \quad x_{0}^{2} \neq q^{m}
$$


For any $\alpha=x_{0}+x_{1} \mathrm{i}+x_{2} \mathrm{j}+x_{3} \mathrm{k} \in \mathbf{H}(\mathbf{Z})$ with $N(\alpha)$ equal to $t$, set

$$
g=\frac{1}{\sqrt{t}}\left[\begin{array}{cc}
x_{0}+x_{1} \mathrm{i} & x_{2}+x_{3} \mathrm{i} \\
-x_{2}+x_{3} \mathrm{i} & x_{0}-x_{1} \mathrm{i}
\end{array}\right] \in G
$$

The image under the homomorphism of $\tilde{g}_{1}, \ldots, \overline{\tilde{g}_{k}}$ yields a set $g_{1}, g_{1}^{-1}, \ldots, g_{k}, g_{k}^{-1}$ in $G$. According to (38) above, $\left\langle g_{1}, \ldots, g_{k}\right\rangle$ is a free group. Note that under this correspondence

$$
\text { trace } \tilde{g}=2 x_{0} / \sqrt{t}
$$

We proceed to estimate the number fo words $\omega$ in $g_{1}, \ldots, g_{k}^{-1}$ of length $m$ with $r_{\omega}$ close to 0 or $\pi$. According to (38) and (39) such a word $\omega$ corresponds to a solution

$$
x_{0}^{2}+x_{1}^{2}+x_{2}^{2}+x_{3}^{2}=q^{m}, \quad x_{0}^{2} \neq q^{m}
$$

and

$$
\sin r_{\omega}=\sqrt{q^{m}-x_{0}^{2}} / q^{m / 2}
$$

From this (32) is clearly satisfied and also

$$
\begin{aligned}
& \#\left\{\omega:|\omega|=m, r_{\omega} \in\left[0, N^{-1+\delta}\right]\right\} \ll \\
& \#\left\{x_{0}^{2}+x_{1}^{2}+x_{2}^{2}+x_{3}^{2}=q^{m}, \sqrt{q^{m}-x_{0}^{2}} / q^{m / 2} \leq N^{-1+\delta}\right\} \ll \\
& \#\left\{x_{0}^{2}+x_{1}^{2}+x_{2}^{2}+x_{3}^{2}=q^{m} ; q^{m / 2}\left(1-N^{2 \delta-2}\right) \leq x_{0}<q^{m / 2}\right\}= \\
& \sum_{\substack{q^{m / 2}\left(1-N^{2 \delta-2},\right)^{1 / 2} \\
\leq x_{0}<q^{m / 2}}} r_{3}\left(q^{m}-x_{0}^{2}\right),
\end{aligned}
$$

where $r_{3}(m)$ is the number of representations of $m$ as a sum of three squares.

From elementary number theory [HW] we have a bound $r_{3}(m)=$ $O_{\varepsilon}\left(m^{1 / 2+\varepsilon}\right)$ for any $\varepsilon>0$. Hence the above sum is

$$
\begin{gathered}
\ll q^{\varepsilon m} \sum_{q^{m / 2}\left(1-N^{2 \delta-2}\right)^{1 / 2}} \leq x_{0}<q^{m / 2} \sqrt{q^{m}-x_{0}^{2}} \ll \\
\ll q^{\varepsilon m}\left(1+\int_{q^{m / 2}\left(1-N^{2 \delta-2}\right)^{1 / 2}}^{q^{m / 2}} \sqrt{q^{m}-t^{2}} d t\right) \ll \\
\ll q^{\varepsilon m}\left(1+q^{m} \int_{0}^{N^{-1+\delta}} \sin ^{2} \theta d \theta\right) \ll \\
\ll q^{\varepsilon m}\left(1+q^{m} / N^{3-3 \delta}\right)
\end{gathered}
$$


Summing this for $m \leq n$ yields

$$
\#\left\{\omega:|\omega| \leq n, r_{\omega} \in\left[0, N^{-1+\delta}\right]\right\} \ll q^{\varepsilon n}\left(1+\frac{q^{n}}{N^{3-3 \delta}}\right)
$$

Note that if $p=q$ (which is the largest set of such $g_{j}$ 's that we can choose) then (41) gives the expected bound (35).

Continuing the analysis as above we get

$$
\sum_{e^{\xi_{j}, N_{0} \geq p^{b}}} 1 \ll_{\varepsilon, \delta} p^{-n b}\left(N_{0}^{2} n+p^{-n / 2} N_{0}^{2} q^{\varepsilon n}\left(1+\frac{q^{n}}{N_{0}^{3-3 \delta}}\right)\right)
$$

Now assume that $p>q^{4 / 5}$ and choose $n$ even satisfying

$$
N_{0}^{3} \leq\left(\frac{q}{\sqrt{p}}\right)^{n} \leq\left(\frac{q}{\sqrt{p}}\right)^{2} N_{0}^{3}
$$

For $N_{0}$ large enough and $p^{b}>q^{2 / 3} / p^{1 / 3}$ we will have the right hand side of (42) being less than 1 . It follows that for $\tilde{\varepsilon}>0$ and $N_{0}$ large enough

$$
\left|\lambda_{j}\left(\widehat{z}\left(\pi_{N_{0}}\right)\right)\right| \leq p^{1 / 2}\left(\frac{q^{2 / 3}}{p^{1 / 3}}+\frac{p^{1 / 3}}{q^{2 / 3}}\right)+\tilde{\varepsilon}
$$

and since $p>q^{4 / 5}$ the latter is less than $p+1=2 k$. This completes the proof of the Theorem 1.1.

\section{Density of states}

Let $z=\sum x_{g} g \in \mathbf{R}[G]$ and assume that $z=z^{*}$. Set

$$
\mu_{N}(z)=\frac{1}{N+1} \sum_{j=0}^{N} \delta_{\lambda_{j}\left(\widetilde{z}\left(\pi_{N}\right)\right)}
$$

Proposition 4.1. There are measures $v^{\text {even }}(z)$ and $v^{\text {odd }}(z)$ such that

$$
\begin{aligned}
& \lim _{\substack{N \rightarrow \infty \\
N \text { even }}} \mu_{N}(z)=v^{\text {even }}(z), \\
& \lim _{\substack{N \rightarrow \infty \\
N \text { odd }}} \mu_{N}(z)=v^{\text {odd }}(z) .
\end{aligned}
$$

(the convergence being in the sense of integration against any continuous function). 
Proof. Fix an integer $m \geq 0$. Then

$$
z^{m}=\sum_{g_{1}, \ldots, g_{m} \in \operatorname{supp}(z)} x_{g_{1}} x_{g_{2}} \ldots x_{g_{m}} g_{1} g_{2} \ldots g_{m}
$$

Hence

$$
\operatorname{trace}\left(\widehat{z^{m}}\left(\pi_{N}\right)\right)=\sum_{g_{1}, \ldots, g_{m}} x_{g_{1}} x_{g_{2}} \ldots x_{g_{m}} \chi_{\pi_{N}}\left(g_{1} g_{2} \ldots g_{m}\right)
$$

or

$$
\mu_{N}(z)\left(x^{m}\right)=\sum_{g_{1}, \ldots, g_{m}} x_{g_{1}} x_{g_{2}} \ldots x_{g_{m}} \chi_{\pi_{N}}\left(g_{1} g_{2} \ldots g_{m}\right) /(N+1)
$$

Now $\chi_{\pi_{N}}(g)=\sin (N+1) \alpha /(\sin \alpha)$ if $g$ is conjugate to $\left[\begin{array}{cc}\mathrm{e}(\alpha) & 0 \\ 0 & \mathrm{e}(-\alpha)\end{array}\right]$. In particular

$$
\begin{aligned}
& \lim _{\substack{N \rightarrow \infty \\
N \text { even }}} \frac{\chi_{\pi_{N}}(g)}{N+1}= \begin{cases}1, & \text { if } g= \pm e, \\
0, & \text { otherwise. }\end{cases} \\
& \lim _{\substack{N \rightarrow \infty \\
N \text { odd }}} \frac{\chi_{\pi_{N}}(g)}{N+1}= \begin{cases}1, & \text { if } g=e, \\
-1, & \text { if } g=-e, \\
0, & \text { otherwise. }\end{cases}
\end{aligned}
$$

Hence

$$
\lim _{\substack{N \rightarrow \infty \\ N \text { even }}} \mu_{\pi_{N}}(z)\left(x^{m}\right):=C_{m}^{e v e n}(z)=\sum_{\substack{g_{1} \ldots, g_{m} \\ g_{1} \ldots, m= \pm e}} x_{g_{1}} \ldots x_{g_{m}}
$$

and

$$
\begin{gathered}
\lim _{\substack{N \rightarrow \infty \\
N \text { odd }}} \mu_{\pi_{N}}(z)\left(x^{m}\right):=C_{m}^{o d d}(z)= \\
\sum_{\substack{g_{1}, \ldots, g_{m} \\
g_{1} \ldots g_{m}=e}} x_{g_{1}} \ldots x_{g_{m}}-\sum_{\substack{g_{1} \ldots, g_{m} \\
g_{1} \ldots . . m=-e}} x_{g_{1}} \ldots x_{g_{m}}
\end{gathered}
$$

Thus for any continuous $f, \mu_{N}(z)(f) \rightarrow v^{\text {even }}(f)$ as $N \rightarrow \infty$ through even values and $\mu_{N}(z)(f) \rightarrow v^{\text {odd }}(f)$ as $N \rightarrow \infty$ through odd values. Here $v^{\text {even }}(z)$ and $v^{\text {odd }}(z)$ are the probability measures (whose existence is clear) and whose moments are given by (45) and (46).

Of special interest are elements of the form

$$
z_{g_{1}, \ldots, g_{k}}=g_{1}+g_{1}^{-1}+g_{2}+g_{2}^{-1}+\ldots+g_{k}+g_{k}^{-1} .
$$


In this case the measures $v^{\text {even }}(z)$ and $v^{\text {odd }}(z)$ can be interpreted as the spectral measures for the random walk on $\Gamma_{z}$ with respect to the symmetric set of generators $g_{1}, g_{1}^{-1}, g_{2}, g_{2}^{-1}, \ldots, g_{k}, g_{k}^{-1}$ (see Kesten [Kest]). It follows from [Kest] that supp $v^{\text {even }}(z)$ and supp $v^{\text {odd }}(z)$ are contained in $[-2 \sqrt{2 k-1}, 2 \sqrt{2 k-1}]$ iff $\Gamma_{z}$ is a free group and in this case

$$
v^{\text {even }}(z)=v^{\text {odd }}(z)=\frac{\sqrt{2 k-1-t^{2} / 4}}{2 \pi k\left(1-(t / 2 k)^{2}\right)} d t .
$$

Moreover Kesten shows that the support of $v^{\text {even }}(z)$ and $v^{\text {odd }}(z)$ are contained in $(-2 k, 2 k)$ iff $\Gamma_{z}$ is not amenable. Hence it is only for $z$ with $\Gamma_{z}$ not amenable that $z$ can have a gap.

We next examine the speed of convergence in Proposition 4.1. This will depend on the diophantine properties of $g_{1}, \ldots, g_{k} \in G$ (we use this terminology to draw an analogy with diophantine approximation).

Definition 4.2. For $k \geq 2$, we say that $g_{1}, g_{2}, \ldots, g_{k} \in G$ are diophantine (or satisfy a diophantine condition) if there is $B=B\left(g_{1}, \ldots, g_{k}\right)>0$ such that for any $m \geq 1$ and a word $R_{m}$ in $g_{1}, g_{2}, \ldots, g_{k}$ of length $m$ with $R_{m} \neq \pm e$ we have

$$
\left\|R_{m} \pm e\right\| \geq B^{-m}
$$

Here

$$
\left\|\left[\begin{array}{ll}
a & b \\
c & d
\end{array}\right]\right\| \|^{2}=|a|^{2}+|b|^{2}+|c|^{2}+|d|^{2} .
$$

Note that for $g \in G$ we have

$$
\|g \pm e\|^{2}=2|\operatorname{trace}(g) \mp 2| .
$$

For example if $\Gamma_{\left(g_{1}, \ldots, g_{k}\right)}$ is finite then $g_{1}, \ldots, g_{k}$ are diophantine, however we are mainly interested in the case that $\Gamma_{\left(g_{1}, \ldots, g_{k}\right)}$ is free. In this case it follows by a pigeon hole argument similar to Dirichlet's that for any $m \geq 1$ there always is a word $R \neq \pm e$ in $g_{1}, g_{1}^{-1}, \ldots, g_{k}, g_{k}^{-1}$ of length at most $m$ satisfying

$$
\|R-e\| \leq \frac{10}{(2 k-1)^{m / 6}}
$$

(here and elsewhere we assume that $k \geq 2$ ). This shows that the exponential behavior in the definition of diophantine is the appropriate one.

As was first exploited by Hausdorff [Hau] the relation

$$
R_{m}\left(g_{1}, g_{1}^{-1}, \ldots, g_{k}, g_{k}^{-1}\right)=e
$$


where $R$ is a reduced word of length $m \geq 1$ is not satisfied identically in $G^{(k)}$. Hence the sets

$$
V\left(R_{m}\right):=\left\{\left(g_{1}, \ldots, g_{k}\right) \mid R_{m}(g)=e\right\}
$$

are of codimension at least one in $G^{(k)}$. It follows that $\cup_{m \geq 1} V\left(R_{m}\right)$ is of zero measure in $G^{(k)}$ and also it is of the first Baire category in $G^{(k)}$. Thus the generic $\left(g_{1}, \ldots, g_{k}\right) \in G^{(k)}$ (in both senses) generates the free group. Now the set of $\left(g_{1}, \ldots, g_{k}\right) \in G^{(k)}$ for which $\left\langle g_{1}, \ldots, g_{k}\right\rangle$ is not free is clearly dense in $G^{(k)}$ so it follows easily that the set of $\left(g_{1}, \ldots, g_{k}\right) \in G^{(k)}$ which are not diophantine is of the second (Baire) category in $G^{(k)}$. That is to say the topologically generic $\left(g_{1}, \ldots, g_{k}\right)$ is free but not diophantine. On the other hand we expect that in the measure sense the generic $\left(g_{1}, \ldots, g_{k}\right)$ is diophantine. For elements with algebraic number entries we have:

Proposition 4.3. Let $g_{1}, \ldots, g_{k} \in G \cap M_{2}(\overline{\mathbf{Q}})$. Then $g_{1}, \ldots, g_{k}$ are diophantine.

Proof. Since the set $\left\{g_{1}, \ldots, g_{k}\right\}$ is finite we have $g_{j} \in M_{2}(K)$ where $K$ is a finite extension of $\mathbf{Q}$ of degree $d$ say. We can find a rational integer $N \geq 1$ such that

$$
N g_{j} \in M_{2}\left(\mathcal{O}_{K}\right)
$$

where $\mathcal{O}_{K}$ is the ring of integers of $K$. Choose $M \geq N$ large enough so that for each of the $d$ embeddings $\sigma$ of $K$ into $\mathbf{C}$ we have

$$
\left\|\sigma\left(N g_{j}\right)\right\| \leq M, \quad j=1, \ldots, k .
$$

If

$$
R=g_{j_{1}} g_{j_{2}} \ldots g_{j_{n}}
$$

is a word of length $n$ in the $g$ 's then

$$
N^{n} R \in M_{2}\left(\mathcal{O}_{K}\right)
$$

Set $h=N^{n} R \pm N^{n} e=N^{n}(R \pm e), h \in M_{2}\left(\mathcal{O}_{K}\right)$ and if $h \neq 0$, say $h_{i j} \neq 0$ then

$$
\left|\sigma\left(h_{i j}\right)\right| \leq\left\|\sigma\left(N^{n} R\right) \pm \sigma\left(N^{n} e\right)\right\| \leq 2 M^{n}
$$

Also

$$
h_{i j} \prod_{\sigma \neq I d} \sigma\left(h_{i j}\right) \in \mathbf{Z}
$$

and is not zero, so we have

$$
\left|h_{i j}\right| \geq \frac{1}{(2 M)^{n d}}
$$


Hence

$$
\|R \pm e\| \geq \frac{1}{\left((2 M)^{d} N\right)^{n}}
$$

Choose $B=(2 M)^{d} N$, then $g_{1}, \ldots, g_{k}$ is diophantine with this choice of $B$.

Our main result about the distribution $\mu_{N}(z)$ for diophantine $z$ is Theorem 1.3.

Proof of Theorem 1.3. The proof of Proposition 4.1 together with the diophantine condition leads to the following. There exists a constant $A=A(z)$ such that for $m \geq 1$,

$$
\begin{cases}\mu_{N}(z)\left(x^{m}\right)=v^{\text {even }}(z)\left(x^{m}\right)+O\left(A^{m} / N\right), & N \text { even }, \\ \mu_{N}(z)\left(x^{m}\right)=v^{\text {odd }}(z)\left(x^{m}\right)+O\left(A^{m} / N\right), & N \text { odd },\end{cases}
$$

the implied constants in (53) being universal (that is independent of $m$ and $N)$. The passage from (53) to the discrepancy bounds is a straightforward analysis of suitable approximation of characteristic functions of intervals by polynomials; we give the details below.

We choose a large constant $K$ and make a change of variables:

$$
\lambda_{j}=K \cos \theta_{j}, \quad 0 \leq \theta_{j} \leq \pi .
$$

We next define new measures $\tilde{\mu}_{N}$ by

$$
\tilde{\mu}_{N}=\sum_{j=0}^{N} \frac{1}{2}\left(\delta_{\theta_{j}}+\delta_{-\theta_{j}}\right) .
$$

The corresponding limit measures as $N \rightarrow \infty$ are denoted by $\widetilde{v}^{\text {even }}, \widetilde{v}^{\text {odd }}$; they will both be called $\widetilde{v}_{\infty}$. The definitions insure that all the measures are even in $\theta$.

Choosing $K$ large enough, we may assume that the measures $\tilde{\mu}_{N}$ and $\widetilde{v}_{\infty}$ are supported in $(-3 \pi / 4,-\pi / 4) \cup(\pi / 4,3 \pi / 4)$. Given $I \subset(-\pi, \pi)$, we want to estimate $\left|\left(\widetilde{\mu}_{N}-\widetilde{v}_{\infty}\right)(I)\right|$. Let $\chi_{I}$ be the characteristic function of the interval $I$. For every $m \in \mathbf{N}$ there exist trigonometric polynomials $S_{m}^{+}(\theta)$ and $S_{m}^{-}(\theta)$ of degree $m$ (which depend on $I$ ), called Selberg polynomials, such that $([\mathrm{Sel}, \mathrm{V}])$

$$
S_{m}^{-}(\theta) \leq \chi_{I}(\theta) \leq S_{m}^{+}(\theta), \int_{-\pi}^{\pi}\left(S_{m}^{+}(\theta)-S_{m}^{-}(\theta)\right) d \theta=O(1 / m),
$$

and the constants are independent of $I$. The degree $m$ of the polynomials will be chosen later. 
By positivity,

$$
\int S_{m}^{-}(\theta) d \widetilde{\mu}_{N} \leq \tilde{\mu}_{N}\left(\chi_{I}(\theta)\right) \leq \int S_{m}^{+}(\theta) d \widetilde{\mu}_{N},
$$

and similar inequalities hold for $\widetilde{v}_{\infty}$ in place of $\widetilde{\mu}_{N}$.

We next remark that since the measures $\widetilde{\mu}_{N}$ and $\widetilde{v}_{\infty}$ are even in $\theta$,

$$
\int S_{m}^{ \pm}\left(d \tilde{\mu}_{N}-d \widetilde{v}_{\infty}\right)=\int S_{m, e v e n}^{ \pm}\left(d \tilde{\mu}_{N}-d \widetilde{v}_{\infty}\right)
$$

where $S_{m, e v e n}^{ \pm}$is the even part of $S_{m}^{ \pm}$.

We make another substitution $y=\cos \theta$. We denote the measures $\tilde{\mu}_{N}$ (respectively, $\widetilde{v}_{\infty}$ ) in the new coordinates by corresponding measures by $\xi_{N}$ (respectively, $\xi_{\infty}$ ). To prove Theorem 1.3 , it suffices to estimate the discrepancy $D\left(\xi_{N}, \xi_{\infty}\right)$.

The functions $S_{m, \text { even }}^{ \pm}(\theta)$ become polynomials of degree $m$ in $y$ which we denote by $f_{m}^{ \pm}(y)$. It is easy to show (cf. [V]) that there exists a constant $B>0$, which does not depend on the interval $I$, such that the coefficients of $f_{m}$ 's grow slower than $B^{m}$. That fact and the bounds (53) imply that for a sufficiently small $c>0$ and for $m=c \ln N$,

$$
\int f_{m}^{ \pm}(y)\left(d \xi_{N}(y)-d \xi_{\infty}(y)\right) \ll 1 /(\ln N) .
$$

To finish the proof, it suffices to show that for $m=c \ln N$,

$$
\int_{\operatorname{supp}\left(\xi_{\infty}\right)}\left(f_{m}^{+}(y)-f_{m}^{-}(y)\right) d \xi_{\infty}(y) \ll 1 /(\ln N) .
$$

By assumptions on $v^{\text {even }}$ and $v^{\text {odd }}$ (which remain valid for $\xi_{\infty}$ ) it suffices to establish (57) for the measure $d y$ instead of $d \xi_{\infty}(y)$.

We note that

$$
\int_{\operatorname{supp}\left(\xi_{\infty}\right)}\left(f_{m}^{+}(y)-f_{m}^{-}(y)\right) d y<\int_{\operatorname{supp}\left(\xi_{\infty}\right)} \frac{f_{m}^{+}(y)-f_{m}^{-}(y)}{\sqrt{1-y^{2}}} d y
$$

(the last integral is well-defined $\operatorname{since} \operatorname{supp}\left(\xi_{\infty}\right) \subset\{|y|<1 / \sqrt{2}\}$ ). The last integral by (56) and a change of variables is equal to

$$
\int_{-\pi}^{\pi}\left(S_{m}^{+}(\theta)-S_{m}^{-}(\theta)\right) d \theta
$$

Accordingly, the bound (55) implies (57) and finishes the proof of Theorem 1.3. 
Remark 4.4. As pointed out above the generic (in topology) $g_{1}, \ldots, g_{k}$ is not diophantine and so does not satisfy the assumptions of Theorem 1.3. In fact in view of Kesten's Theorem above about the support of $v(z)$ and $\Gamma_{z}$ being free, together with the density of $\left(g_{1}, \ldots, g_{k}\right)$ which are not free, it follows by the usual Baire category argument (cf. [LPS]) that Theorem 1.3 fails for topologically generic $z\left(g_{1}, \ldots, g_{k}\right)$.

On the other hand the numerical experiments of Sect. 5 indicate that for the generic in measure $\left(g_{1}, \ldots, g_{k}\right), k \geq 3, \operatorname{spec}\left(\widehat{z}\left(\pi_{N}\right)\right)$ behaves like that of a member of the corresponding random matrix ensemble. If this is so then Theorem 1.3 for such a generic $z$ is very far from the true upper bound which would be of order $(\log N) / N$ rather than $1 / \log N$. For the case of the specific Ramanujan elements $z_{p}$ of Sect. 3 we now establish a lower bound for $D$.

Proof of Theorem 1.4. A $z_{p}$ as above satisfies $\operatorname{spec}\left(\widehat{z}_{p}\left(\pi_{N}\right)\right) \subset[-2 \sqrt{p}$, $2 \sqrt{p}$ ] for $N \geq 1$. Our aim is to show that $D\left(\mu_{N}\left(z_{p}\right), v\left(z_{p}\right)\right)$ is large at least on a subsequence $N_{j} \rightarrow \infty$. The measure $v\left(z_{p}\right)$ is the Kesten measure defined in (47).

One checks that the discrepancy is invariant under continuous monotone changes of variable in the eigenvalue parameter. Since $\lambda_{j, N} \in$ $[-2 \sqrt{p}, 2 \sqrt{p}]$ is is convenient to use the variable $\theta_{j, N} \in[0, \pi]$ where

$$
2 \sqrt{p} \cos \theta_{j, N}=\lambda_{j, N}, \quad 0 \leq j \leq N ; N \geq 1
$$

Set

$$
\tilde{\mu}_{N}\left(z_{p}\right)=\frac{1}{N+1} \sum_{j=0}^{N} \delta_{\theta_{j, N}}
$$

which is a probability measure on $[0, \pi]$. Let $\widetilde{v}_{p}$ be the corresponding limit of the $\tilde{\mu}_{N}\left(z_{p}\right)$ as $N \rightarrow \infty$. For $n \geq 3$ let

$$
I_{n, N}=(N+1) \int_{0}^{\pi}\left(\frac{\sin (n+1) t}{\sin t}-\frac{\sin (n-1) t}{p \sin t}\right) d \widetilde{\mu}_{N}(t) .
$$

Note that

$$
\int_{0}^{\pi}\left(\frac{\sin (n+1) t}{\sin t}-\frac{\sin (n-1) t}{p \sin t}\right) d \widetilde{v}_{p}(t)=0 .
$$

Thus an integration by parts in (59) yields

$$
(N+1)^{-1}\left|I_{n, N}\right| \leq 2 n^{2} D\left(\tilde{\mu}_{N}, \widetilde{v}_{p}\right)
$$


We will use (61) to give a lower bound for $D\left(\widetilde{\mu}_{N}, \widetilde{v}_{p}\right)$. According to (27) we have

$$
I_{n, N}=p^{-n / 2} \sum_{|\omega|=n}^{\prime} \frac{\sin (N+1) r_{\omega}}{\sin r_{\omega}} .
$$

For the element $z_{p}$ we saw in Sect. 3 that the right hand side of (62) may be expressed as

$$
p^{-n / 2} \sum_{|x|<p^{n / 2}} v(n, x) \frac{\sin \left((N+1) \beta_{x, n}\right)}{\sin \left(\beta_{x, n}\right)}
$$

where $v(n, x)$ is the multiplicity of reduced words of length $n$ with the trace equal to $x / p^{n / 2}$, that is

$$
\cos \beta_{x, n}=\frac{x}{p^{n / 2}}, \quad x \in \mathbf{Z},|x|<p^{n / 2} .
$$

From the case $N=0$ we have

$$
p^{n-1}(1+p)=\sum_{|x|<p^{n / 2}} v(n, x)
$$

Now rewrite (63) as

$$
I_{n, N}=\sum_{|x|<p^{n / 2}} \frac{v(x, n)}{\sqrt{p^{n}-x^{2}}} \sin \left((N+1) \beta_{x, n}\right) .
$$

Let $f \in \mathcal{S}(\mathbf{R})$ be even, $f \geq 0, f(0)=0$ and $\int_{-\infty}^{\infty} f(x) d x=1$. Assume further that supp $\hat{f} \subset(-1,1)$. Consider

$$
\begin{aligned}
& \sum_{N=0}^{\infty} f\left(\frac{N+1}{N_{0}}\right)\left|I_{n, N}\right|^{2}=\frac{1}{2} \sum_{x} \frac{(v(x, m))^{2}}{p^{n}-x^{2}} \sum_{m=-\infty}^{\infty} f\left(\frac{m}{N_{0}}\right)\left(\sin m \beta_{x, n}\right)^{2} \\
& +\frac{1}{2} \sum_{x \neq x^{\prime}} \frac{(v(x, n)) v\left(x, n^{\prime}\right)}{\sqrt{\left(p^{n}-x^{2}\right)\left(p^{n}-\left(x^{\prime}\right)^{2}\right)}} \sum_{m=-\infty}^{\infty} f\left(\frac{m}{N_{0}}\right) \sin \left(m \beta_{x, n}\right) \sin \left(m \beta_{x^{\prime}, n}\right)
\end{aligned}
$$

$$
\begin{aligned}
& \text { If } x \neq x^{\prime} \\
& \qquad \frac{1}{p^{n / 2}} \leq\left|\frac{x-x^{\prime}}{p^{n / 2}}\right|=\left|\cos \beta_{x, N}-\cos \beta_{x^{\prime}, N}\right| \leq\left|\beta_{x, N}-\beta_{x^{\prime}, N}\right|
\end{aligned}
$$

while

$$
\beta_{x, N}+\beta_{x^{\prime}, N}<2 \pi-1 / p^{n / 2}
$$


Thus applying Poisson summation in (67) together with supp $\hat{f} \subset(-1,1)$ we see that for $N_{0}>2 p^{n / 2}$ the second term in (67) vanishes while the first equals

$$
\frac{N_{0}}{4}\left(\int_{-\infty}^{\infty} f(x) d x\right) \sum_{|x|<p^{n / 2}} \frac{(v(x, n))^{2}}{p^{n}-x^{2}} .
$$

That is we have

$$
\sum_{N=0}^{\infty} f\left(\frac{N+1}{N_{0}}\right)\left|I_{n, N}\right|^{2}=\frac{N_{0}}{4} \sum_{|x|<p^{n / 2}} \frac{(v(x, n))^{2}}{p^{n}-x^{2}}
$$

if $p^{n / 2}<N_{0} / 2$. On the other hand from (65) we have

$$
p^{n} \leq \sum_{|x|<p^{n / 2}} v(x, n) \leq\left(\sum_{|x|<p^{n / 2}} \frac{v^{2}(x, n)}{p^{n}-x^{2}}\right)^{1 / 2}\left(\sum_{|x|<p^{n / 2}}\left(p^{n}-x^{2}\right)\right)^{1 / 2}
$$

Hence

$$
\sum_{|x|<p^{n / 2}} \frac{v^{2}(x, n)}{p^{n}-x^{2}} \geq \frac{p^{n / 2}}{4} .
$$

That is for $p^{n / 2}<N_{0} / 2$,

$$
\sum_{N=0}^{\infty} f\left(\frac{N+1}{N_{0}}\right)\left|I_{n, N}\right|^{2} \geq \frac{N_{0} p^{n / 2}}{16}
$$

Choose $n$ so that

$$
\frac{N_{0}}{2 \sqrt{p}}<p^{n / 2}<\frac{N_{0}}{2} .
$$

We then have

$$
\frac{1}{N_{0}} \sum_{N=0}^{\infty} f\left(\frac{N+1}{N_{0}}\right)\left|I_{n, N}\right|^{2} \gg N_{0}
$$

and hence from (61) that

$$
\frac{1}{N_{0}} \sum_{N=0}^{\infty} f\left(\frac{N+1}{N_{0}}\right)\left|D\left(\tilde{\mu}_{N}, \widetilde{v}_{p}\right)\right|^{2} \gg \frac{N_{0}^{-1}}{\left(\log N_{0}\right)^{4}}
$$

In particular this means that there is a subsequence $N_{j} \rightarrow \infty$ such that

$$
D\left(\tilde{\mu}_{N_{j}}, \widetilde{v}_{p}\right) \gg \frac{N_{j}^{-1 / 2}}{\left(\log N_{j}\right)^{2}} .
$$

This establishes the required lower bound for the discrepancy claimed in Theorem 1.4. 


\section{Numerical results}

We report on some numerical computations of $\operatorname{spec}\left(\widehat{z}\left(\pi_{N}\right)\right)$ for certain $z$ and for $N$ of size of about 1000. As pointed out in the Introduction, if $z=g_{1}+g_{1}^{-1}+g_{2}+g_{2}^{-1}$ and is in generic position there is a nontrivial $h \in G$ commuting with $z$ (as elements of $\mathbf{R}[G]$ ). This impacts the statistics of the distributions of the eigenvalues of $\widehat{z}\left(\pi_{N}\right)$. To avoid this symmetry we consider in this section elements of the form $g_{1}+g_{1}^{-1}+g_{2}+g_{2}^{-1}+g_{3}+g_{3}^{-1}$. For such a $z$ with $\left(g_{1}, g_{2}, g_{3}\right)$ generic it appears ${ }^{1}$ that centralizing subalgebra of $z$ in $\mathbf{R}[G]$ is $\mathbf{R}[z]$. The numerics below also confirm that the generic such $g$ is "desymmetrized". The precise $z$ 's which we consider are

(I) $z$ chosen at random (i.e. $g_{1}, g_{2}, g_{3}$ chosen at random).

(II) $z$ is the Ramanujan element $z_{5}$, that is

$$
g_{1}=A=\frac{1}{\sqrt{5}}\left[\begin{array}{cc}
1+2 i & 0 \\
0 & 1-2 i
\end{array}\right], \quad g_{2}=B=\frac{1}{\sqrt{5}}\left[\begin{array}{cc}
1 & 2 \\
-2 & 1
\end{array}\right],
$$

and

$$
g_{3}=C=\frac{1}{\sqrt{5}}\left[\begin{array}{cc}
1 & 2 i \\
2 i & 1
\end{array}\right]
$$

(III) $z$ the element $\tilde{z}$ defined after Theorem 1.1 with $q=7$,

$$
\widetilde{A}=\frac{1}{\sqrt{7}}\left[\begin{array}{cc}
2-i & 1+i \\
-1+i & 2+i
\end{array}\right], \quad \widetilde{B}=\frac{1}{\sqrt{7}}\left[\begin{array}{cc}
2-i & -1+i \\
1+i & 2+i
\end{array}\right],
$$

and

$$
\widetilde{C}=\frac{1}{\sqrt{7}}\left[\begin{array}{cc}
2+i & -1+i \\
1+i & 2-i
\end{array}\right]
$$

In all these cases $\Gamma_{z}$ is a free group on 3 generators so that the density of states is given by (47) with $k=3$.

We first describe the method of computation. A direct computation of the matrix of $\widehat{z}\left(\pi_{N}\right)$ using the basis (11) (or variants thereof using orthogonal polynomials) turn out to be too time consuming and inaccurate when $N$ is of size 1000. What is much better is to use matrix exponentiation as follows. The Lie algebra su(2) of SU(2) is spanned by the vectors

$$
X_{1}=\left[\begin{array}{cc}
i & 0 \\
0 & -i
\end{array}\right], X_{2}=\left[\begin{array}{cc}
0 & 1 \\
-1 & 0
\end{array}\right], X_{3}=\left[\begin{array}{ll}
0 & i \\
i & 0
\end{array}\right] .
$$

For $X \in \operatorname{su}(2)$ and $\pi$ a representation of $G$ (in particular $\pi_{N}$ ) we have the relation

$$
\pi(\exp X)=\exp (d \pi(X))
$$

\footnotetext{
1 This question will be addressed in more detail in a separate paper.
} 
The matrix of $d \pi_{N}\left(X_{j}\right), j=1,2,3$ may be computed explicitly from the action (1) and the basis of $W_{N+1}$ normalized according to (12). For $j=0,1, \ldots, N$ let

$$
\widehat{e}_{j}=\frac{x^{j} y^{N-j}}{\sqrt{j !(N-j) !}}
$$

Then

$$
\left\{\begin{array}{l}
d \pi_{N}\left(X_{1}\right)\left(\widehat{e}_{j}\right)=i(2 j-N) \widehat{e}_{j}, \\
d \pi_{N}\left(X_{2}\right)\left(\widehat{e}_{j}\right)=-\sqrt{j(N-j+1)} \widehat{e}_{j-1}+\sqrt{(N-j)(j+1)} \widehat{e}_{j+1}, \\
d \pi_{N}\left(X_{3}\right)\left(\widehat{e}_{j}\right)=i \sqrt{j(N-j+1)} \widehat{e}_{j-1}+i \sqrt{(N-j)(j+1)} \widehat{e}_{j+1} .
\end{array}\right.
$$

Thus $d \pi_{N}\left(X_{j}\right)$ is skew-Hermitian with $d \pi_{N}\left(X_{1}\right)$ diagonal and $d \pi_{N}\left(X_{2}\right)$, $d \pi_{N}\left(X_{3}\right)$ tridiagonal. The exponentials of these matrices were computed using MATLAB which appeals to the Pade approximation with scaling and squaring algorithm (see [GV, method 11.3.1]). A nice discussion of the subtleties of computing such exponentials of matrices is given in [MV]. Once the exponential is computed we use (75) to obtain $\widehat{z}\left(\pi_{N}\right)$ in this basis. The eigenvalues of this matrix were then computed, again using MATLAB.

We turn to an analysis of the results and their interpretation. The first data concerns the density of states. For the random $z=g_{1}+g_{1}^{-1}+g_{2}+$ $g_{2}^{-1}+g_{3}+g_{3}^{-1}$ (that is to say we chose $X^{(j)}=\xi_{1} X_{1}+\xi_{2} X_{2}+\xi_{3} X_{3}$ at random for $j=1,2,3$ and then used these to get $\left.g_{j}=\exp \left(X^{(j)}\right)\right)$ the distributions $\mu_{N}(z)$ were computed for various $N$ 's. The graphs all looked alike and in Fig. 1 the case $N=1004$ with a histogram of $\mu_{1004}(z)$ is drawn against the density (47). The fit is excellent and is consistent with the discrepancy being $O((\log N) / N)$.

On the other hand the same experiment was carried out for the Ramanujan element $z_{5}$ (note that $A, B, C$ in $z_{5}$ are very conveniently expressed as $\exp \left(\xi X_{1}\right), \exp \left(\xi X_{2}\right), \exp \left(\xi X_{3}\right)$ with $\left.\cos \xi=3 / 5\right)$ and the result is displayed in Fig. 2. The discrepancy is much larger and is consistent with being of size $1 / \sqrt{N}$ as proven in Theorem 1.3.

The second set of data concerns the consecutive spacings distribution between the eigenvalues of $\widehat{z}\left(\pi_{N}\right)$. As is apparent from Fig. 1 the density of eigenvalues is essentially constant between -3 and 3 . To use more of the data set one straightens or unfolds the eigenvalues by the change of variables

$$
F(x)=\int_{-2 \sqrt{5}}^{x} \frac{6 \sqrt{20-t^{2}}}{2 \pi\left(36-t^{2}\right)} d t
$$

which renders the density uniform. The histogram of the distribution for the resulting consecutive spacings between these straightened numbers (renormalized to have mean 1) was then computed. 


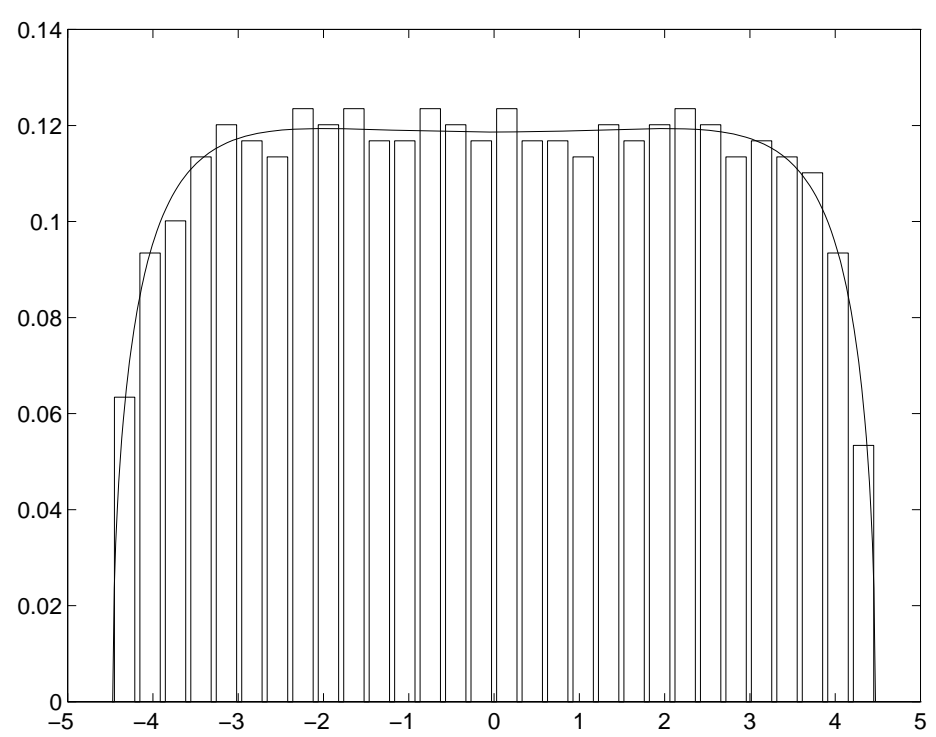

Fig. 1. Density for a random $z, N=1004, k=3$, vs. Kesten's measure

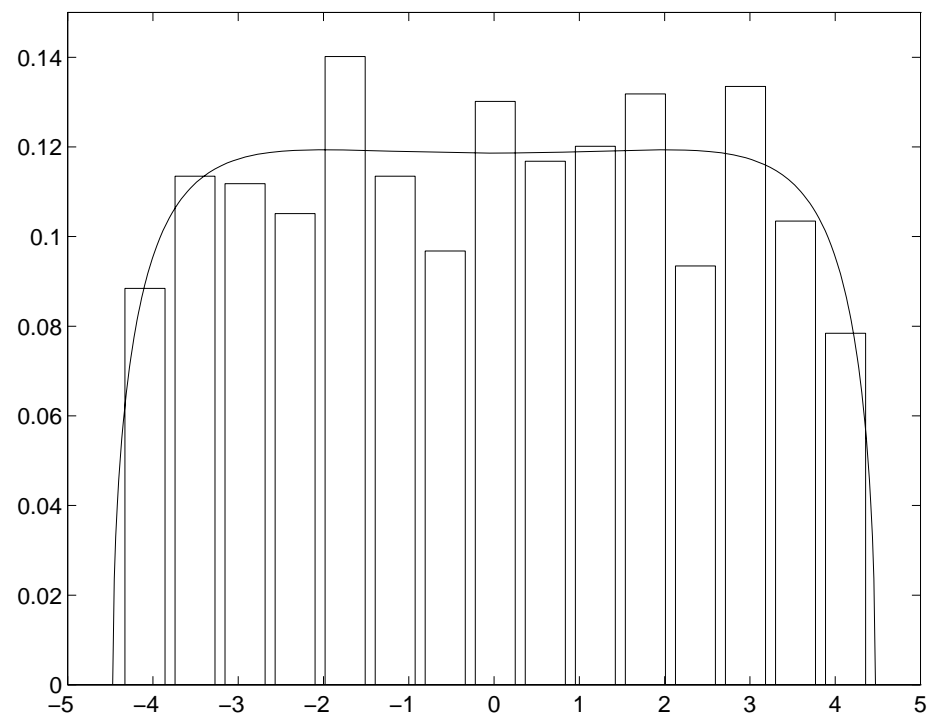

Fig. 2. Density for $A, B, C$ of (II), $N=1004$, vs. Kesten's measure

For a random $z$ and 10 values of $N$ in the range [700,1100] the results were all alike. In Fig. 3 the case $N=1099$ is displayed against the Gaussian Symplectic Ensemble prediction [M] (actually we used the Wigner surmise which is a very good approximation to the latter). Note that in the case of $N$ odd we know that the spectrum of $\widehat{z}\left(\pi_{N}\right)$ comes in doublets and we took 


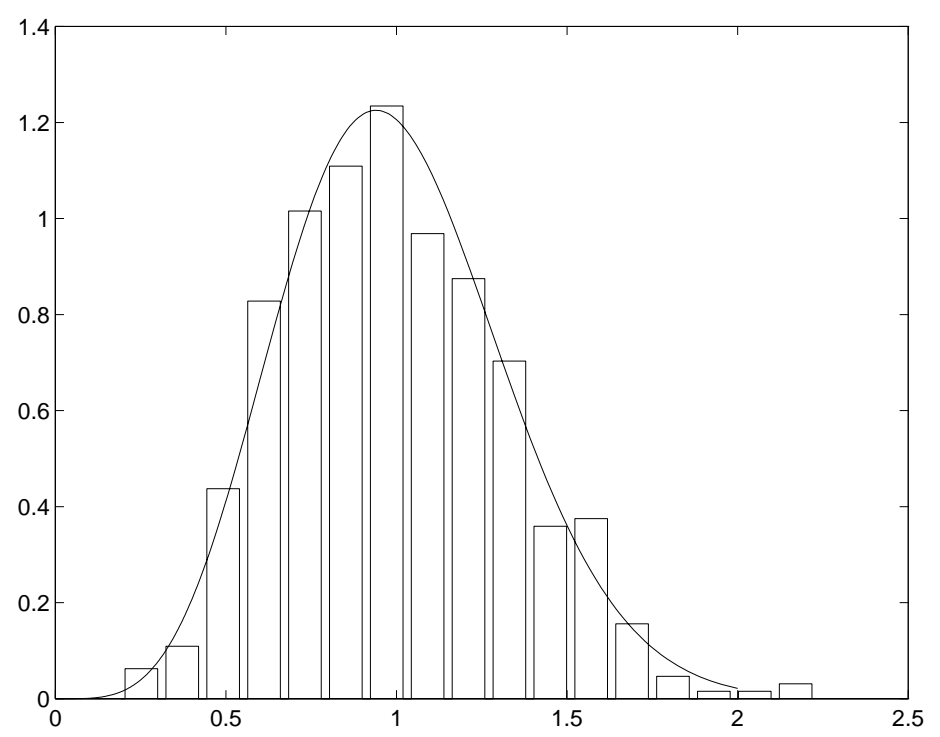

Fig. 3. Spacings for a random $z, N=1099$, vs. GSE

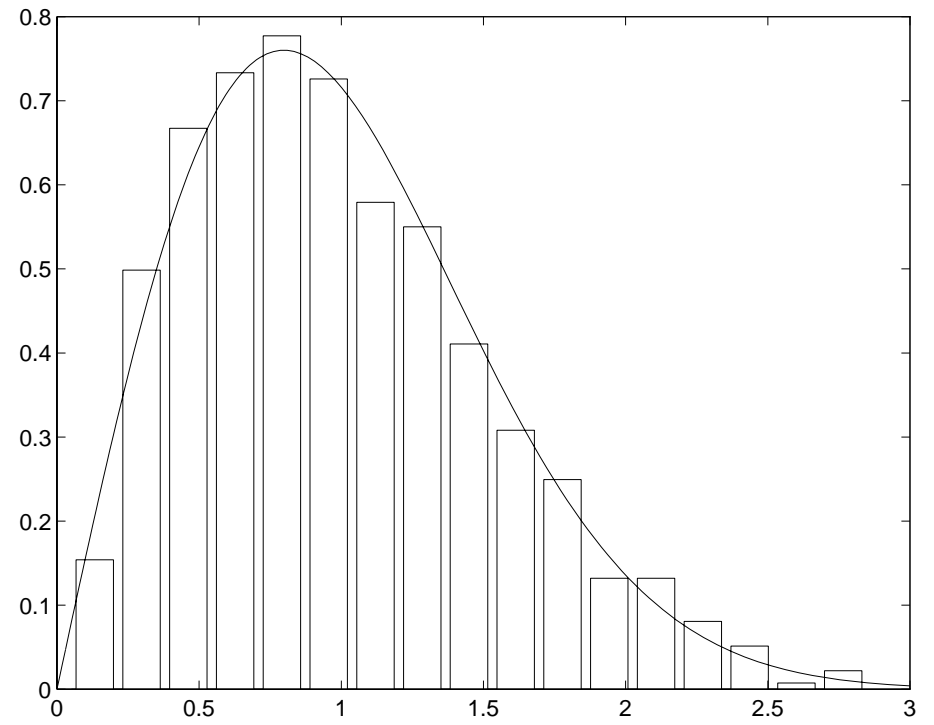

Fig. 4. Spacings for a random $z, N=1004$, vs. GOE

one eigenvalue out of each of these. The fit is very good (theoretically we might expect the discrepancy to be of order $1 / \sqrt{N}$ ).

A similar calculation for $N$ even gave GOE statistics. A typical case being the histogram in Fig. 4 for $N=1004$ which is compared with the GOE Wigner surmise. 
For the case of $z_{5}$ the spacings behave very differently. Firstly there is added multiplicity to the eigenvalues. This may be accounted for by symmetry. The subgroup $H$ of $G$ which centralizes $z_{5}$ is isomorphic to the symmetric group $S_{4}$. A convenient geometric way of seeing this is to represent a rotation $R$ in $\mathrm{SO}(3)$ as $\xi=\left(\xi_{1}, \xi_{2}, \xi_{3}\right)$, where $\xi$ is the axis of rotation of $R$ and whose length is the angle of rotation. The direction is chosen so that the rotation is at most $\pi$ about the oriented axis. For $A, B, C$ in (II) above, the $\xi$ representations are $\xi_{A}=(0,0, \tau), \xi_{B}=(0, \tau, 0)$ and $\xi_{C}=(\tau, 0,0)$ with $\cos \tau=3 / 5$. Now if $g_{0}$ corresponds to $\xi$ and $g$ is in $\mathrm{SO}(3)$, then $g^{-1} g_{0} g$ corresponds to $g \xi$. Hence since the vectors $\xi_{A}, \xi_{A^{-1}}, \xi_{B}, \xi_{B^{-1}}, \xi_{C}, \xi_{C^{-1}}$ are the vertices of a regular octahedron, we see that $H$, the rotational symmetry group, is the octahedral group $S_{4}$.

We decompose the action of $H$ on $W_{N+1}$ into irreducible subspaces on each of which $z$ acts as a scalar. The irreducible representations and character table of $S_{4}$ are well known. $\left|S_{4}\right|=24$ and there are five conjugacy classes. The identity element, 6 transpositions $(a b), 3$ elements of order $2(a b)(c d)$, 8 elements $(a b c)$ of order 3 and 6 elements of order $4(a b c d)$. There are two one dimensional irreducible representations $\chi_{0}$ and $\varepsilon$, one two dimensional representation $\theta$ and two three dimensional representations $\psi$ and $\varepsilon \psi$. The character table together with the character $\chi_{N}, N=2 l$ of $\mathrm{SO}(3)$ (using the double cover $\mathrm{SU}(2) \rightarrow \mathrm{SO}(3))$ is given in Table 1.

\section{Table 1.}

\begin{tabular}{|c|c|c|c|c|c|}
\hline & 1 & $(a b)$ & $(a b)(c d)$ & $(a b c)$ & $(a b c d)$ \\
\hline \hline$\chi_{0}$ & 1 & 1 & 1 & 1 & 1 \\
\hline$\varepsilon$ & 1 & -1 & 1 & 1 & -1 \\
\hline$\theta$ & 2 & 0 & 2 & -1 & 0 \\
\hline$\psi$ & 3 & 1 & -1 & 0 & -1 \\
\hline$\varepsilon \psi$ & 3 & -1 & -1 & 0 & 1 \\
\hline$\chi_{2 l}$ & $2 l+1$ & $(-1)^{l}$ & $(-1)^{l}$ & $\varepsilon_{3}(l)$ & $\varepsilon_{4}(l)$ \\
\hline
\end{tabular}

Here

$$
\varepsilon_{3}(l)= \begin{cases}1, & \text { if } l \equiv 0(\bmod 3), \\ 0, & \text { if } l \equiv 1(\bmod 3), \\ -1, & \text { if } l \equiv 2(\bmod 3),\end{cases}
$$

and

$$
\varepsilon_{4}(l)= \begin{cases}1, & \text { if } l \equiv 0,1(\bmod 4), \\ -1, & \text { if } l \equiv 2,3(\bmod 4)\end{cases}
$$


Using the scalar product $\left\langle\chi_{2 l}, \chi_{\sigma}\right\rangle$ we can count the multiplicity $m_{\sigma}$ with which $\sigma$ appears in $W_{2 l+1}$. We get

$$
\begin{aligned}
m_{0} & =\left(2 l+1+9(-1)^{l}+8 \varepsilon_{3}(l)+6 \varepsilon_{4}(l)\right) / 24 \\
m_{\varepsilon} & =\left(2 l+1-3(-1)^{l}+8 \varepsilon_{3}(l)-6 \varepsilon_{4}(l)\right) / 24 \\
m_{\theta} & =\left(2 l+1+3(-1)^{l}-4 \varepsilon_{3}(l)\right) / 12 \\
m_{\psi} & =\left(2 l+1+(-1)^{l}-2 \varepsilon_{4}(l)\right) / 8 \\
m_{\varepsilon \psi} & =\left(2 l+1-3(-1)^{l}+2 \varepsilon_{4}(l)\right) / 8
\end{aligned}
$$

Thus if there are no degeneracies in the spectrum of $\widehat{z}_{5}\left(\pi_{2 l+1}\right)$ beyond what is forced by the symmetry group $H$ then we would have $m_{0}+m_{\varepsilon}$ simple eigenvalues, $m_{\theta}$ eigenvalues of multiplicity two, and $m_{\psi}+m_{\varepsilon \psi}$ eigenvalues of multiplicity three. We checked this for 10 different $l$ 's and found exactly those multiplicities in each case. Note that the multiplicity two part is "pure" and the consecutive spacing distribution for it was computed. The results were all more or less the same. Fig. 5 gives the pure spectrum for three values of $N$ of order 1000 and compares the distribution with $e^{-x} d x$ (i.e. "Poissonian"). Several values of $N$ were taken because the pure part of the spectrum for $N$ of that order is rather small (less than 100).

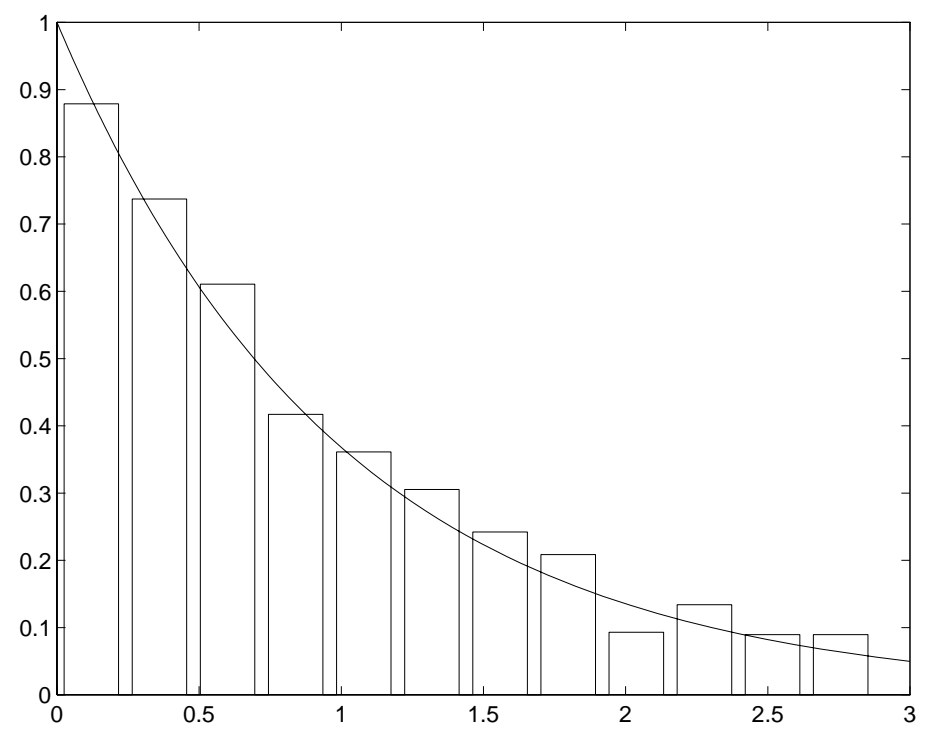

Fig. 5. Spacings for $A, B, C$ of (II), $N \approx 1000$, vs. $e^{-x} d x$ ("pure" part)

Combining all the spectrum for $\widehat{z}_{5}\left(\pi_{2 l+1}\right)$ (taking one eigenvalue out of each multiple set) gives a similar distribution, see Fig. 6. Presumably the 


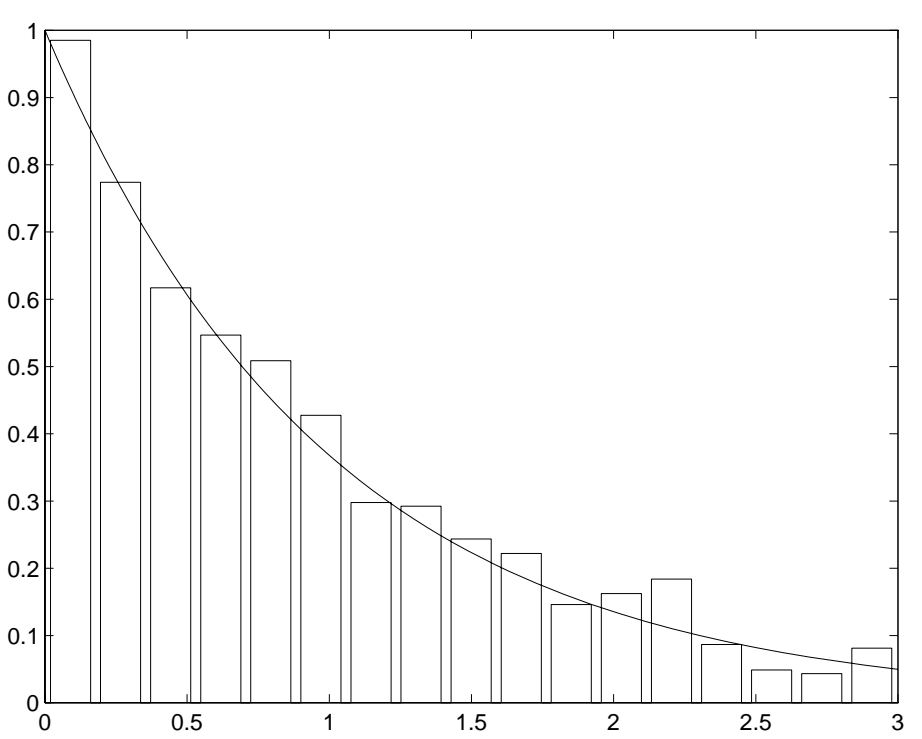

Fig. 6. Spacings for $A, B, C$ of (II), $N=1004$, vs. $e^{-x} d x$ ("combined")

reason being that each "pure" piece is Poissonian and the superposition of independent Poissonians is again Poissonian.

A similar analysis for the element $\tilde{z}$ (cf. (III) above) was carried out. Recall that in Theorem 1.1 we showed that $\left\|\tilde{z}\left(\pi_{N}\right)\right\| \leq 5.83<6$. It turns out that $\tilde{z}$ is not a Ramanujan element since for example $\left\|\tilde{z}\left(\pi_{80}\right)\right\|=$ $4.5842 \ldots>4.4721 \ldots=2 \sqrt{5}$. Again there are degeneracies in the spectrum due to symmetry. This time the $\xi$ vectors corresponding to $\widetilde{A}, \widetilde{B}, \widetilde{C}$ are

$$
\sqrt{\frac{\alpha}{3}}(-1,1,1), \sqrt{\frac{\alpha}{3}}(-1,-1,1), \sqrt{\frac{\alpha}{3}}(1,-1,1)
$$

where $\cos \alpha=1 / 7$. The vectors $\xi_{\widetilde{A}}, \xi_{\widetilde{A}^{-1}}, \xi_{\widetilde{B}}, \xi_{\widetilde{B}^{-1}}, \xi_{\widetilde{C}}, \xi_{\widetilde{C}^{-1}}$ form the three diagonals of the cube with barycenter at the origin. The rotation symmetry group is $S_{3}$ and an analysis as above with its irreducibles (there is one 2-dimensional and two 1-dimensional representations) yields a "pure" part in $W_{2 l+1}$ of multiplicity two. The dimension of this part is $2\left(2 l+1-\varepsilon_{3}(l)\right) / 3$ and the rest of the space corresponds to the 1-dimensional pieces. Again this accounted for all the degeneracy in the spectrum for the l's we checked. The consecutive spacing distribution for the pure multiplicity two part is given in Fig. 7 when $N=912$. Again it was typical of what was found for other $N$ 's. The approximation by GOE is quite good. As for the multiplicity one part of the spectrum, it is not GOE. The reason is that it consists of eigenvalues from both of the 1-dimensional representations which are being superimposed. 


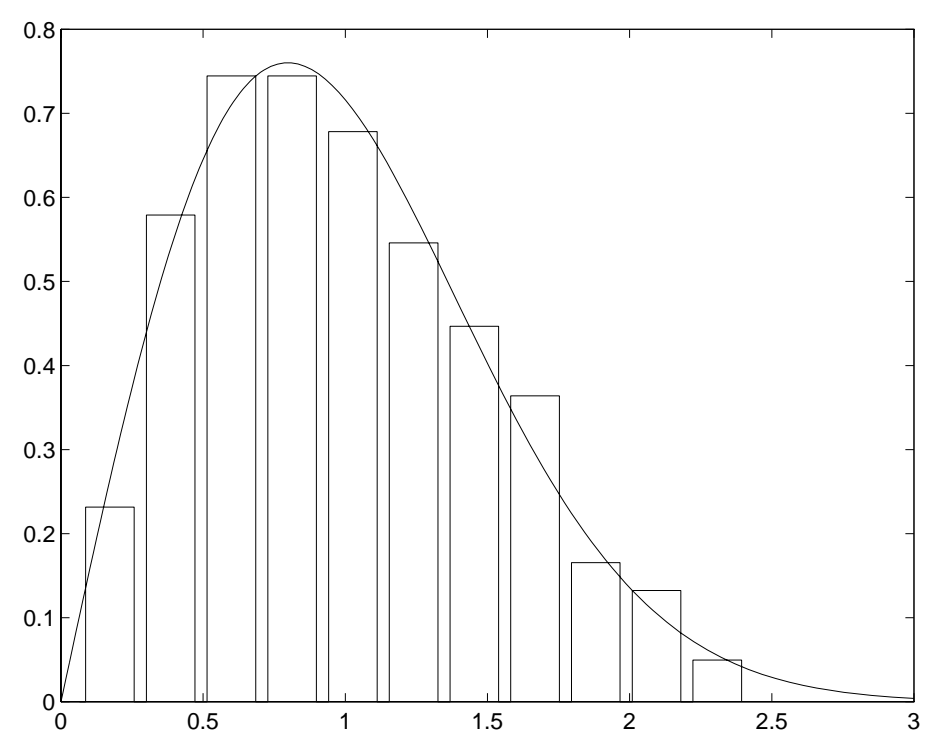

Fig. 7. Spacings for $\widetilde{A}, \widetilde{B}, \widetilde{C}$ of (III), $N=912$, vs. GOE ("pure" part)

\section{References}

[BGGS] E. Bogomolny, B. Georgeot, M.-J. Giannoni, C. Schmit: Chaotic billiards generated by arithmetic groups. Phys. Rev. Lett. 69(10), 1477-1480 (1992)

[BGS] O. Bohigas, M. Giannoni, C. Schmit: Characterization of chaotic quantum spectra and universality of level fluctuation laws. Phys. Rev. Lett. 52(1), 1-4 (1984)

[BSS] J. Bolte, G. Steil, F. Steiner: Arithmetical chaos, violation of universality in energy level statistics. Phys. Rev. Lett. 69(15), 2188-2191 (1992)

[CV] Y. Colin de Verdière: Distribution de points sur une sphère (d'après Lubotzky, Phillips et Sarnak). Astérisque 177/178, 83-93 (1989)

[Dri] V. Drinfeld: Finitely-additive measures on $S^{2}, S^{3}$, invariant with respect to rotations. Functional Anal. Appl. 18, 245-246 (1984)

[GV] G. Golub, C. Van Loan: Matrix computations, 3rd edn. JHU Press 1996

[H] M. Hall: The theory of groups, reprint of the 1968 edn. Chelsea 1976

[HW] G. Hardy, E. Wright: An introduction to the theory of numbers, fifth edn. Oxford University Press, Oxford 1979

[Hau] F. Hausdorff: Grundzüge der Mengenlehre. Leipzig 1914

[Hej] D. Hejhal: The Selberg trace formula for PSL $_{2}$ (R). Vol. I, LNM 548. Springer, Berlin, Heidelberg, New York 1976, Vol. II, LNM 1001. Springer, Berlin, Heidelberg, New York 1983

[Kest] H. Kesten: Symmetric random walks on groups. Trans. AMS 92, 336-354 (1959)

[LPS] A. Lubotzky, R. Phillips, P. Sarnak: Hecke operators and distributing points on $S^{2}$. I. Comm. Pure Appl. Math. 39(S), S149-S186 (1986) II. Comm. Pure Appl. Math. 40(4), 401-420 (1987)

[Lub] A. Lubotzky: Discrete groups, expanding graphs and invariant measures. Birkhauser, Basel 1994

[LW] A. Lubotzky, B. Weiss: Groups and expanders. In: Expanding graphs (Princeton, NJ, 1992), 95-109. DIMACS Ser. Discr. Math. Theor. Comp. Sci. 10, AMS 1993

[LS] W. Luo, P. Sarnak: Number variance for arithmetic hyperbolic surfaces. Commun. Math. Phys. 161, 419-432 (1994)

[M] M. Mehta: Random matrices, 2nd edn. Academic Press, New York 1991 
[MV] C. Moler, C. Van Loan: Nineteen dubious ways to compute the exponential of a matrix. SIAM Rev. 20, 801-836 (1978)

[Sa1] P. Sarnak: Statistical properties of eigenvalues of the Hecke operators. In: Analytic number theory and Diophantine problems (Stillwater, 1984), pp. 321-331. Progr. Math. 70, Birkhauser, Basel 1987

[Sa2] P. Sarnak: Spectra and Eigenfunctions of the Laplacians. In: Partial differential equations and their applications (Toronto, 1995), pp. 261-276. CRM Proc. Lecture Notes 12, AMS, 1997

[Sch] K. Schmidt: Amenability, Kazhdan's property $T$, strong ergodicity and invariant means for ergodic group-actions. Ergodic Theory Dyn. Syst. 1, 223-236 (1981)

[Sel] A. Selberg: Lectures on sieves. In: A. Selberg: Collected papers, Vol. II., pp. 65-247. Springer, Berlin, Heidelberg, New York 1991

[Ser] J-P. Serre: Repartition asymptotique des valeurs propres de l'operateur de Hecke $T_{p}$. JAMS 10, 75-102 (1997)

[Sha] Y. Shalom: Random ergodic theorems, invariant means and unitary representations (preprint)

[V] J. Vaaler: Some extremal functions in Fourier analysis. Bull. AMS 12, 183-216 (1985)

[Wa] S. Wagon: The Banach-Tarski paradox. corr. reprint. Cambridge University Press, Cambridge 1993

[Zel1] S. Zelditch. Index and dynamics of quantized contact transformations. Ann. Inst. Fourier 47, 305-363 (1997)

[Zel2] S. Zelditch. Quantum dynamics from the semiclassical viewpoint (preprint 1997) 